\title{
Coupled-cluster studies of infinite nuclear matter
}

\author{
G. Baardsen, ${ }^{1}$ A. Ekström, ${ }^{1,2}$ G. Hagen,${ }^{3,4}$ and M. Hjorth-Jensen ${ }^{1,2,5}$ \\ ${ }^{1}$ Department of Physics and Center of Mathematics for Applications, University of Oslo, N-0316 Oslo, Norway \\ ${ }^{2}$ National Superconducting Cyclotron Laboratory, Michigan State University, East Lansing, MI 48824, USA \\ ${ }^{3}$ Physics Division, Oak Ridge National Laboratory, Oak Ridge, TN 37831, USA \\ ${ }^{4}$ Department of Physics and Astronomy, University of Tennessee, Knoxville, TN 37996, USA \\ ${ }^{5}$ Department of Physics and Astronomy, Michigan State University, East Lansing, MI 48824, USA
}

(Dated: September 16, 2021)

Background: Coupled-cluster (CC) theory is a widely used many-body method for studying strongly correlated many-fermion systems. It allows for systematic inclusions of complicated manybody correlations beyond a mean-field. Recent applications to finite nuclei have shown that first principle approaches like CC theory can be extended to studies of medium-heavy nuclei, with excellent agreement with experiment. However, CC calculations of properties of infinite nuclear matter are rather few and date back more than 30 years in time.

Purpose: The aim of this work is thus to develop the relevant formalism for performing coupledcluster calculations in nuclear matter and neutron star matter, including thereby important correlations to infinite order in the interaction and testing modern nuclear forces based on chiral effective field theory. Our formalism includes also the exact treatment of the so-called Pauli operator in a partial wave expansion of the equation of state.

Methods: Nuclear and neutron matter calculations are done using a coupled particle-particle and hole-hole ladder approximation. The coupled ladder equations are derived as an approximation of CC theory, leaving out particle-hole and non-linear diagrams from the CC doubles amplitude equation. This study is a first step toward CC calculations for nuclear and neutron matter.

Results: We present results for both symmetric nuclear matter and pure neutron matter employing state-of-the-art nucleon-nucleon interactions based on chiral effective field theory. We employ also the newly optimized chiral interaction [A. Ekström et al., Phys. Rev. Lett. 110, 192502 (2013)] to study infinite nuclear matter. The ladder approximation method and corresponding results are compared with conventional Brueckner-Hartree-Fock theory. The ladder approximation is derived and studied using both exact and angular-averaged Pauli exclusion operators, with angular-averaged input momenta for the single-particle potentials in all caluclations. The inclusion of an exact treatment of the Pauli operators in a partial wave expansion yields corrections of the order of $1.7-2 \%$ of the total energy in symmetric nuclear matter. Similarly, the inclusion of both hole-hole and particle-particle ladders result in corrections of the order $0.7-2 \%$ compared to the approximation with only particle-particle ladders. Including these effects, we get at most almost a $6 \%$ difference between our CC calculation and the standard Brueckner-Hartree-Fock approach.

Conclusions: We have performed CC calculations of symmetric nuclear matter and pure neutron matter including particle-particle and hole-hole diagrams to infinite order using an exact Pauli operator and angular-averaged single-particle energies. The contributions from hole-hole diagrams and exact Pauli operators add important changes to the final energies per particle.

PACS numbers: 21.65.Cd, 21.30.-x, 21.10.-k, 03.75.Ss, 26.60.-c, 26.60.Kp, 21.65.-f

\section{INTRODUCTION}

Nuclear matter is defined as an isotropic system consisting of infinitely many nucleons which interact only by nuclear forces. The Schrödinger equation of this system has been solved approximately using many different $a b$ initio many-body methods $1-8$. As an example, diagrammatic partial summations have been derived from many-body perturbation theory to calculate the binding energy. One approach belonging to this family of methods is the Brueckner-Goldstone (BG) expansion 9, which is a Goldstone expansion where the interaction has been replaced by a well-behaved reaction matrix 10]. The Brueckner-Hartree-Fock (BHF) scheme [9, 11, which is one of the standard methods of nuclear matter theory [6, 12 16, is a first-order approximation in BG theory.
Unfortunately, the BG expansion does not converge very well when using the number of reaction matrices as the order parameter [17]. A more appropriate way to include higher-order correlations is the hole-line expansion [1, where the perturbation truncation is determined by the number of independent hole lines in the the Brueckner-Goldstone diagrams. The two-hole-line approximation is then equivalent to the BHF method. Calculations including up to three-hole-line diagrams indicate that the hole-line expansion converges [13, 18]. Despite encouraging results for the hole-line expansion, it would still be desirable to get a deeper understanding of the accuracy of the many-body methods applied to nuclear matter. Better knowledge about the convergence of the many-body methods in nuclear matter is also necessary to validate the quality of calculations including three- and many-body interactions [19 25]. 
An approach that is related to the above-mentioned perturbative techniques is the coupled-cluster (CC) method [26-31]. As perturbation theory, coupled-cluster theory gives a non-variational and size-extensive method. However, in contrast to many-body perturbation theory, $\mathrm{CC}$ theory sums to infinite order, depending on the level of truncation, selected many-body contributions. It is thus a non-perturbative method. The coupled-cluster truncation is physical in the sense that a so-called $T_{n}$ truncation includes all possible correlations arising from sets of $n$-particle- $n$-hole clusters 32 34. During the last one and a half decade, CC theory has been successfully applied to structure calculations of finite nuclei [35 43]. Inspired by the success of the CC approach in finite nuclei, we hope that CC theory could also provide accurate results for infinite nuclear matter.

Already in the early 1980's, Day and Zabolitzky performed CC calculations for nuclear matter 22 using the Bochum truncation 44, 45. The theory of nuclear interactions has evolved a lot since that time, see for example Ref. [46, with the construction of high-precision potentials exhibiting a $\chi^{2}$ per datum close to one with respect to nucleon-nucleon scattering data 46]. Later, two- and three-body interactions have been developed based on effective field theory (EFT), which is derived using symmetries arising from quantum chromodynamics (QCD) [47]. In addition, soft low-momentum interactions based on renormalization group theory have been introduced 48. It seems therefore necessary to perform new CC calculations for nuclear matter with modern two- and three-body interactions.

In the Bochum truncation scheme [4], the exact CC amplitude equations are approximated by setting all socalled reduced $n$-particle subsystem amplitudes $\chi_{n}$, with $n$ larger than a chosen truncation level, to zero. The justification for using this truncation is that all subsystem amplitudes $\chi_{n}$ become small inside a radius where the interaction may be very strong [44. This truncation scheme therefore ensures that the contribution from particles interacting strongly at short distances becomes small. In their CC calculations for nuclear matter, Day and Zabolitzky included the two- and three-body subsystem amplitudes $\chi_{2}$ and $\chi_{3}$, and incorporated also parts of the four-body amplitude $\chi_{4}[2,18$. Given the computing capacity in the early 1980 s, it was necessary to do further approximations in the CC equations. As is explained in Refs. 2, 18, the authors used angular-averaged Pauli exclusion operators, other averages over angles, and firstorder Taylor expansions to approximate the dependence of the single-particle potential on the $G$-matrix. In addition, they replaced some diagrams by estimates, which were simpler to calculate than the original diagrams [2]. Before this study, Manzke [49] did CC calculations for nuclear matter in the two-body subsystem approximation.

Coupled cluster theory is widely used within the quantum chemistry community 31. A commonly used CC truncation scheme in quantum chemistry is to set all cluster amplitudes in the exponential CC wave function ansatz beyond a given excitation level to zero (see Sec. II A and Refs. [32, 33). These approximations are called for example $\mathrm{CC}$ doubles (CCD), CC singlesdoubles (CCSD), etc. 31, or alternatively SUB $n$ approximations [50. This truncation scheme has been applied in studies of finite nuclei [37, 38, 40 43, mainly using soft interactions. Our aim is to apply the same CC method to studies of the equation of state of symmetric nuclear matter and neutron matter.

The SUB $n$ approximation includes long-range contributions, such as the forward and other ring diagrams, already at the CCD or SUB2 level [50, 51. In contrast, these correlations are included in the Bochum scheme in the subsystem amplitudes $\chi_{3}$ and $\chi_{4}$ [44]. Another difference between the two CC truncation schemes is the treatment of the single-particle state potentials in the energy denominator. In the Bochum CC method, the energy denominator contains single-hole state potentials with summations of particle-particle ladder diagrams to infinite order, whereas the single-particle state potentials are zero in the energy denominator. Instead, the singleparticle state potential terms are part of the $\chi_{3}$ and $\chi_{4}$ subsystem amplitudes [44. In contrast, in the SUBn approximation, which we employ in this work, the energy denominator contains single-particle potentials at the Hartree-Fock level for both particles and holes.

Previously, Freeman [52 has studied the twodimensional electron gas including particle-particle ladder diagrams from the $\mathrm{CC}$ doubles approximation. In a similar way, as a first step toward CC calculations for nuclear matter, we will here include particle-particle and hole-hole correlations at the 2-particle-2-hole, or $T_{2}$, level. In this scheme, only the linear ladder diagrams have been included in the CC $T_{2}$ amplitude equation, whereas the linear particle-hole diagrams and all non-linear diagrams have been neglected. When leaving out certain diagrams of the $T_{2}$ amplitude equation, our scheme may be considered as strictly not a coupled-cluster approximation. However, the method shows a proof of principle of an iterative coupled-cluster numerical scheme, where particleparticle and hole-hole ladder diagrams are coupled and summed to infinite order. The coupled ladder approximation is similar to the Bochum CC method including only the two-body subsystem amplitude $\chi_{2}$, but the singleparticle potentials are different. It ought also to be emphasized that the calculations of Day and Zabolitzky [2] included a larger set of diagrams, and thereby correlations, than the approximation used in this work.

According to the hole-line expansion calculations by Song et al., the contribution of particle-particle diagrams is considerably larger than that of particle-hole diagrams [13. Still, the contribution of particle-hole diagrams is clearly non-negligible in the hole-line expansion [13. The results of Ref. [53] show that ring (particle-hole) diagrams are significant for the binding energy of nuclear matter. The aim is therefore to include all $T_{2}$ diagrams in a future $\mathrm{CC}$ calculation to get a proper $\mathrm{CC}$ approximation at the 
$T_{2}$ level.

Summation of particle-particle and hole-hole ladder diagrams is also a common approximation in self-consistent Green's function (SCGF) theory [4. The SCGF ladder approximation has been extensively applied to studies of nuclear and neutron matter [54 [58], lately including either three-body interactions or density dependent two-body operators arising from three-body interactions [8, 19. In the SCGF ladder approximation, the energy denominator contains self-consistently solved complete off-shell self-energies including both particle-particle and hole-hole ladder diagrams. As the SCGF method, the CC ladder approximation also treats particle and hole interactions symmetrically, but from the definition of the $\mathrm{CC}$ equations it follows that the single-particle potentials occur in the energy denominator only up to the HartreeFock level.

Another similar method is the particle-particle and hole-hole ring diagram approximation [59 62, where the particle-particle and hole-hole diagrams are derived from Green's function theory, and a momentum model space is used to avoid poles in the energy denominator. The binding energy obtained in this approximation depends however on the model space momentum cutoff [61. In the particle-particle and hole-hole ring diagram method the authors employed the standard angular-average approximations in order to decrease the computational complexity of the calculations [59].

In the present work, we will analyze the partial wave expansion of the equation of state using an exact treatment of the intermediate states, avoiding thereby the standard angle-average approximation of Pauli exclusion operators 12. Finally, we perform calculations of the above systems using modern nucleon-nucleon interactions based on chiral perturbation theory to next-tonext-to-leading order (NNLO) 63] and next-to-next-tonext-to-leading order $\left(\mathrm{N}^{3} \mathrm{LO}\right) 64$.

After these introductory remarks, we present our formalism in the next three sections, followed subsequently by our results and discussion thereof in Sec. IV] Concluding remarks and perspectives are presented in Sec. V.

\section{FORMALISM: MANY-BODY METHODS}

The general form of the Hamiltonian operator of infinite nuclear matter is

$$
\begin{aligned}
\hat{H} & =\hat{K}+\hat{V}_{N N}+\hat{V}_{N N N}+\ldots \\
& =-\frac{\hbar^{2}}{2 M} \sum_{i=1}^{A} \nabla_{i}^{2}+\sum_{i<j}^{A} \hat{v}_{N N}\left(\mathbf{r}_{i}, \mathbf{r}_{j}\right) \\
& +\sum_{i<j<k}^{A} \hat{v}_{N N N}\left(\mathbf{r}_{i}, \mathbf{r}_{j}, \mathbf{r}_{k}\right)+\ldots,
\end{aligned}
$$

where $A$ is the number of nucleons, $\hat{K}$ is the kinetic energy operator, $\hat{V}_{N N}$ is a two-particle interaction operator,
$\hat{V}_{N N N}$ is a three-particle interaction operator, $M$ is the nucleon mass, and $\mathbf{r}_{l}$ is the position vector of particle $l$. In this paper, we neglect $n$-body interactions for $n$ larger than two and define the Hamiltonian operator as

$$
\hat{H}=\hat{K}+\hat{V}=-\frac{\hbar^{2}}{2 M} \sum_{i=1}^{A} \nabla_{i}^{2}+\sum_{i<j}^{A} \hat{v}\left(r_{i j}\right),
$$

where $\hat{v}$ is a two-body interaction and $r_{i j}=\left|\mathbf{r}_{i}-\mathbf{r}_{j}\right|$.

In our calculations, we use the nucleon-nucleon interaction of Ref. 64. This interaction model is given by an $\mathrm{N}^{3} \mathrm{LO}$ approximation of chiral perturbation theory. Nuclear interactions based on effective field theory have the advantage that two- and many-body interactions can be derived in a mutually consistent way [47. Furthermore, we present also results obtained with a recent nucleonnucleon interaction at order NNLO in chiral perturbation theory. This interaction results from an optimizationbased re-parameterization to the available body of experimental data using the model-based, derivative-free optimization algorithm POUNDerS developed at Argonne National Laboratory 65. The resulting new chiral interaction, labeled $\mathrm{NNLO}_{\text {opt }}$ hereafter, exhibits a $\chi^{2}$ per datum close to one for laboratory scattering-energies below approximately $125 \mathrm{MeV}$ in the two-body proton-proton and neutron-proton channels, see Ref. 63. for further details. In the $A=3$ and $A=4$ nucleon systems, this interaction gives binding energies that differ by $20 \mathrm{keV}$ and $45 \mathrm{keV}$ from the experimental values, respectively. Thus, the contributions of three-nucleon forces appear smaller than for previous parametrizations of chiral interactions.

We model infinite nuclear matter as a system of $A$ interacting nucleons confined by a cubic box potential. The cubic box boundary condition together with the free nucleon Hamiltonian equation

$$
-\frac{\hbar^{2}}{2 M} \nabla^{2} \varphi(\mathbf{r})=\varepsilon \varphi(\mathbf{r})
$$

gives the plane wave eigenfunctions $\varphi_{\mathbf{k}}(\mathbf{r})=e^{i \mathbf{k} \cdot \mathbf{r}} / \sqrt{\Omega}$ and eigenenergies $\varepsilon_{\mathbf{k}}=\hbar^{2} k^{2} /(2 M)$. Here $\hbar \mathbf{k}$ is the momentum, $\mathbf{r}$ is the position coordinate, and $\Omega$ is the volume of the box. We therefore use plane waves as our single-particle basis, from which the Slater determinants are constructed.

\section{A. Coupled ladder approximation}

In this subsection, the coupled ladder equations will be derived as an approximation of the coupled-cluster method. The coupled-cluster formalism is presented in a momentum basis. In the general expressions, we omit spin and isospin degrees of freedom.

In coupled-cluster theory, the $A$-fermion state vector is expressed using the exponential ansatz

$$
|\Psi\rangle \equiv e^{\hat{T}}\left|\Phi_{0}\right\rangle
$$


where $\left|\Phi_{0}\right\rangle$ is the uncorrelated free Fermi vacuum, and the cluster operator $\hat{T}$ is defined as the sum

$$
\hat{T}=\sum_{m=1}^{A} \hat{T}_{m},
$$

of $m$-particle $m$-hole excitation operators

$$
\begin{aligned}
\hat{T}_{m} & =\left(\frac{1}{m !}\right)^{2} \sum_{\substack{\mathbf{k}_{i_{1}}, \ldots, \mathbf{k}_{i_{m}} \\
\mathbf{k}_{a_{1}} \ldots, \mathbf{k}_{a_{m}}}} t_{\mathbf{k}_{i_{1}} \ldots \mathbf{k}_{i_{m}}}^{\mathbf{k}_{a_{1}} \ldots \mathbf{k}_{a_{m}}} \\
& \times c_{\mathbf{k}_{a_{1}}}^{\dagger} \ldots c_{\mathbf{k}_{a_{m}}}^{\dagger} c_{\mathbf{k}_{i_{m}}} \ldots c_{\mathbf{k}_{i_{1}}} .
\end{aligned}
$$

We label single-particle states occupied in the Fermi vacuum determinant $\Phi_{0}$ (holes) by $i, j, k, \ldots$ and excited states of the same single-particle basis (particles) by $a, b, c$, and so on. Indices $p, q, r, \ldots$ are used to label single-particle states that may be either holes or particles. The operators $c^{\dagger}$ and $c$ are fermion creation and annihilation operators, respectively.

Given that the single-particle basis is complete, the $A$ particle Schrödinger equation can be written equivalently as the CC energy equation

$$
\left\langle\Phi_{0}\left|e^{-\hat{T}} \hat{H} e^{\hat{T}}\right| \Phi_{0}\right\rangle=E,
$$

where the cluster operator $\hat{T}$ is obtained from the corresponding set of CC amplitude equations

$$
\left\langle\Phi_{\mathbf{k}_{i_{i}} \mathbf{k}_{i_{2}} \ldots \mathbf{k}_{i_{k}}}^{\mathbf{k}_{a_{1}} \mathbf{k}_{a_{2} \ldots \mathbf{k}_{a_{k}}}}\left|e^{-\hat{T}} \hat{H} e^{\hat{T}}\right| \Phi_{0}\right\rangle=0
$$

for $k=1,2,3, \ldots, A$. Here we have used the notation

$$
\begin{aligned}
\left|\Phi_{\mathbf{k}_{i_{1}} \mathbf{k}_{i_{2}} \ldots \mathbf{k}_{i_{k}}}^{\mathbf{k}_{a_{1}} \mathbf{k}_{a_{2}} \ldots \mathbf{k}_{a_{k}}}\right\rangle & \equiv c_{\mathbf{k}_{a_{1}}}^{\dagger} c_{\mathbf{k}_{a_{2}}}^{\dagger} \ldots c_{\mathbf{k}_{a_{k}}}^{\dagger} \\
& \times c_{\mathbf{k}_{i_{k}}}^{\dagger} \ldots c_{\mathbf{k}_{i_{2}}} c_{\mathbf{k}_{i_{1}}}\left|\Phi_{0}\right\rangle,
\end{aligned}
$$

which means that the bra vector in Eq. (4) is a $k$-particle $k$-hole excitation of the Fermi vacuum state.

In almost all practical calculations, except for some very simple model systems, it is necessary to do a truncation both in the cluster operator $\hat{T}$ and in the singleparticle basis. To derive the ladder expansion, we need only the approximation $\hat{T} \approx \hat{T}_{2}$, which is commonly called the CC doubles approximation (CCD). In fact, the $\hat{T}_{1}$ operator is found to vanish for infinite nuclear and neutron matter 44. By symmetry considerations, the total momentum of the system of nucleons is zero. Both the kinetic energy operator $\hat{K}$ and the total Hamiltonian $\hat{H}$ are assumed to be diagonal in total momentum $\mathbf{K}$. Hence, both the reference state $\Phi_{0}$ and the correlated ground state $\Psi$ must be eigenfunctions of the operator $\hat{\mathbf{K}}$ with the corresponding eigenvalue $\mathbf{K}=\mathbf{0}[10$.

Using abstract vectors and a momentum single-particle basis, the CC ansatz can be written as

$$
\begin{aligned}
\left|\Psi_{C C}\right\rangle & =\left|\Phi_{0}\right\rangle+\sum_{\mathbf{k}_{i} \mathbf{k}_{a}} t_{\mathbf{k}_{i}}^{\mathbf{k}_{a}}\left|\Phi_{\mathbf{k}_{i}}^{\mathbf{k}_{a}}\right\rangle+\frac{1}{4} \sum_{\mathbf{k}_{i} \mathbf{k}_{j} \mathbf{k}_{a} \mathbf{k}_{b}} t_{\mathbf{k}_{i} \mathbf{k}_{j}}^{\mathbf{k}_{a} \mathbf{k}_{b}}\left|\Phi_{\mathbf{k}_{i} \mathbf{k}_{j}}^{\mathbf{k}_{a} \mathbf{k}_{b}}\right\rangle \\
& +\frac{1}{2} \sum_{\mathbf{k}_{i} \mathbf{k}_{a}} \sum_{\mathbf{k}_{j} \mathbf{k}_{b}} t_{\mathbf{k}_{i}}^{\mathbf{k}_{\mathbf{a}}} t_{\mathbf{k}_{j}}^{\mathbf{k}_{b}}\left|\Phi_{\mathbf{k}_{i} \mathbf{k}_{j}}^{\mathbf{k}_{a} \mathbf{k}_{b}}\right\rangle+\ldots
\end{aligned}
$$

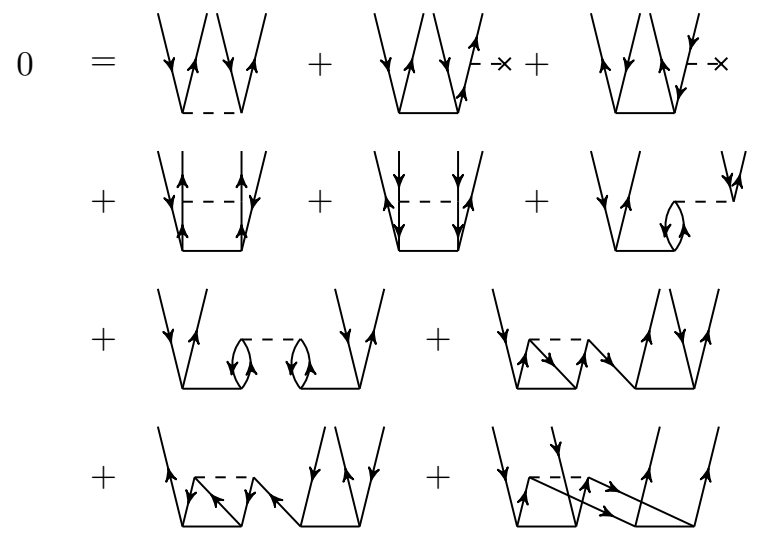

FIG. 1. Diagrammatic representation of the $\hat{T}_{2}$ amplitude equation in the coupled-cluster doubles approximation. The coupled particle-particle and hole-hole ladder equations (PPHH-LAD) are obtained by leaving out the sixth diagram, which has summation over one particle and one hole state, and all the non-linear diagrams, that is, the four last diagrams. The particle-particle ladder equations (PP-LAD) is otherwise equal to the PPHH-LAD equations, apart from that in the former case also the fifth diagram is neglected. The two-particle interaction is illustrated by a dashed line and the $t$-amplitude by a solid line. The dashed line with a cross at one vertex represents the Fock operator. Lines with arrows pointing upwards represent particles whereas lines with arrows pointing downwards represent holes. The interaction and $t$-amplitude are assumed to be antisymmetric.

where $\mathbf{k}_{m}$ is the momentum of the single-particle state $m$. From Eq. (6) and the conditions

$$
\hat{\mathbf{K}}\left|\Phi_{0}\right\rangle=\mathbf{0}\left|\Phi_{0}\right\rangle,
$$

and

$$
\hat{\mathbf{K}}\left|\Psi_{C C}\right\rangle=\mathbf{0}\left|\Psi_{C C}\right\rangle,
$$

it follows that

$$
\hat{\mathbf{K}} t_{\mathbf{k}_{i}}^{\mathbf{k}_{a}}\left|\Phi_{\mathbf{k}_{i}}^{\mathbf{k}_{a}}\right\rangle=\left(\mathbf{k}_{a}-\mathbf{k}_{i}\right) t_{\mathbf{k}_{i}}^{\mathbf{k}_{a}}\left|\Phi_{\mathbf{k}_{i}}^{\mathbf{k}_{a}}\right\rangle
$$

and

$$
\hat{\mathbf{K}} t_{\mathbf{k}_{i} \mathbf{k}_{j}}^{\mathbf{k}_{a} \mathbf{k}_{b}}\left|\Phi_{\mathbf{k}_{i} \mathbf{k}_{j}}^{\mathbf{k}_{a} \mathbf{k}_{b}}\right\rangle=\left(\mathbf{k}_{a}+\mathbf{k}_{b}-\mathbf{k}_{i}-\mathbf{k}_{j}\right) t_{\mathbf{k}_{i} \mathbf{k}_{j}}^{\mathbf{k}_{a} \mathbf{k}_{b}}\left|\Phi_{\mathbf{k}_{i} \mathbf{k}_{j}}^{\mathbf{k}_{a} \mathbf{k}_{b}}\right\rangle .
$$

Since by definition $\left|\mathbf{k}_{a}\right|>\left|\mathbf{k}_{i}\right|$, the CC exponential ansatz becomes an eigenfunction of the operator $\hat{\mathbf{K}}$ with the eigenvalue $\mathbf{0}$ only if all coefficients $t_{\mathbf{k}_{i}}^{\mathbf{k}_{a}}$ are zero and the restriction

$$
\mathbf{k}_{a}+\mathbf{k}_{b}=\mathbf{k}_{i}+\mathbf{k}_{j},
$$

is fulfilled. This implies that the operator $\hat{T}_{1}$ is zero. In the same way, the contribution of $\hat{T}_{1}$ vanishes in coupledcluster calculations for the three-dimensional electron gas [50].

The CCD $\hat{T}_{2}$ amplitude equation can be written in laboratory momentum coordinates as, see for example 
Ref. 33],

$$
\begin{aligned}
0 & =\left\langle\mathbf{k}_{a} \mathbf{k}_{b}|v| \mathbf{k}_{i} \mathbf{k}_{j}\right\rangle \\
& +P\left(\mathbf{k}_{a} \mathbf{k}_{b}\right) \sum_{\mathbf{k}_{c}}\left\langle\mathbf{k}_{b}|f| \mathbf{k}_{c}\right\rangle\left\langle\mathbf{k}_{a} \mathbf{k}_{c}|t| \mathbf{k}_{i} \mathbf{k}_{j}\right\rangle \\
& -P\left(\mathbf{k}_{i} \mathbf{k}_{j}\right) \sum_{\mathbf{k}_{k}}\left\langle\mathbf{k}_{a} \mathbf{k}_{b}|t| \mathbf{k}_{i} \mathbf{k}_{k}\right\rangle\left\langle\mathbf{k}_{k}|f| \mathbf{k}_{j}\right\rangle \\
& +\frac{1}{2} \sum_{\mathbf{k}_{c} \mathbf{k}_{d}}\left\langle\mathbf{k}_{a} \mathbf{k}_{b}|v| \mathbf{k}_{c} \mathbf{k}_{d}\right\rangle\left\langle\mathbf{k}_{c} \mathbf{k}_{d}|t| \mathbf{k}_{i} \mathbf{k}_{j}\right\rangle \\
& +\frac{1}{2} \sum_{\mathbf{k}_{k} \mathbf{k}_{l}}\left\langle\mathbf{k}_{a} \mathbf{k}_{b}|t| \mathbf{k}_{k} \mathbf{k}_{l}\right\rangle\left\langle\mathbf{k}_{k} \mathbf{k}_{l}|v| \mathbf{k}_{i} \mathbf{k}_{j}\right\rangle \\
& +P\left(\mathbf{k}_{i} \mathbf{k}_{j}\right) P\left(\mathbf{k}_{a} \mathbf{k}_{b}\right) \sum_{\mathbf{k}_{k} \mathbf{k}_{c}}\left\langle\mathbf{k}_{a} \mathbf{k}_{c}|t| \mathbf{k}_{i} \mathbf{k}_{k}\right\rangle\left\langle\mathbf{k}_{k} \mathbf{k}_{b}|v| \mathbf{k}_{c} \mathbf{k}_{j}\right\rangle \\
& +\frac{1}{2} P\left(\mathbf{k}_{i} \mathbf{k}_{j}\right) P\left(\mathbf{k}_{a} \mathbf{k}_{b}\right) \sum_{\mathbf{k}_{k} \mathbf{k}_{l} \mathbf{k}_{c} \mathbf{k}_{d}}\left\langle\mathbf{k}_{k} \mathbf{k}_{l}|v| \mathbf{k}_{c} \mathbf{k}_{d}\right\rangle \\
& \times\left\langle\mathbf{k}_{a} \mathbf{k}_{c}|t| \mathbf{k}_{i} \mathbf{k}_{k}\right\rangle\left\langle\mathbf{k}_{d} \mathbf{k}_{b}|t| \mathbf{k}_{l} \mathbf{k}_{j}\right\rangle \\
& -P\left(\mathbf{k}_{i} \mathbf{k}_{j}\right) \frac{1}{2} \sum_{\mathbf{k}_{k} \mathbf{k}_{l} \mathbf{k}_{c} \mathbf{k}_{d}}\left\langle\mathbf{k}_{k} \mathbf{k}_{l}|v| \mathbf{k}_{c} \mathbf{k}_{d}\right\rangle \\
& \times\left\langle\mathbf{k}_{a} \mathbf{k}_{b}|t| \mathbf{k}_{i} \mathbf{k}_{k}\right\rangle\left\langle\mathbf{k}_{c} \mathbf{k}_{d}|t| \mathbf{k}_{j} \mathbf{k}_{l}\right\rangle \\
& -P\left(\mathbf{k}_{a} \mathbf{k}_{b}\right) \frac{1}{2} \sum_{\mathbf{k}_{k} \mathbf{k}_{l} \mathbf{k}_{c} \mathbf{k}_{d}}\left\langle\mathbf{k}_{k} \mathbf{k}_{l}|v| \mathbf{k}_{c} \mathbf{k}_{d}\right\rangle \\
& \times\left\langle\mathbf{k}_{a} \mathbf{k}_{c}|t| \mathbf{k}_{i} \mathbf{k}_{j}\right\rangle\left\langle\mathbf{k}_{b} \mathbf{d}|t| \mathbf{k}_{k} \mathbf{k}_{l}\right\rangle \\
& +\frac{1}{4} \sum_{\mathbf{k}_{k} \mathbf{k}_{l} \mathbf{k}_{c} \mathbf{k}_{d}}\left\langle\mathbf{k}_{k} \mathbf{k}_{l}|v| \mathbf{k}_{c} \mathbf{k}_{d}\right\rangle \\
& \times\left\langle\mathbf{k}_{c} \mathbf{k}_{d}|t| \mathbf{k}_{i} \mathbf{k}_{j}\right\rangle\left\langle\mathbf{k}_{a} \mathbf{k}_{b}|t| \mathbf{k}_{k} \mathbf{k}_{l}\right\rangle \\
&
\end{aligned}
$$

where all two-body matrix elements are antisymmetrized and $P(p q)$ is a permutation operator that operates on a general function $y(p, q)$ according to

$$
P(p q) y(p, q)=y(p, q)-y(q, p) .
$$

The Fock operator is defined by

$$
\left\langle\mathbf{k}_{p}|f| \mathbf{k}_{q}\right\rangle=\left\langle\mathbf{k}_{p}\left|h_{0}\right| \mathbf{k}_{q}\right\rangle+\sum_{i}\left\langle\mathbf{k}_{p} \mathbf{k}_{i}|v| \mathbf{k}_{q} \mathbf{k}_{i}\right\rangle,
$$

where the sinlge-particle kinetic energy operator $h_{0}$ is $k^{2} /(2 M)$ in momentum space. From the fact that the two-particle interaction conserves the total momentum, it follows that the Fock operator is diagonal in momentum basis. This also means that the plane wave basis is a Hartree-Fock basis for infinite nuclear matter and, as is well known, the Hartree-Fock energy for nuclear matter is simply the same as the ground state energy in first order many-body perturbation theory $(\mathrm{MBPT}(1))$ 66. The CC $\hat{T}_{2}$ amplitude equation from Eq. (7) is given in diagrammatic representation in Fig. 1. We use diagrammatic rules as defined in Ref. [33.

The particle-particle and hole-hole ladder approximation (PPHH-LAD) is obtained by leaving out from the CCD amplitude equation all nonlinear terms, as well as

$$
E_{C C D}=0-x+0--\circ+\vartheta^{--}
$$

FIG. 2. The CCD energy equation, given in terms of diagrams. The two-particle interaction is given by a dashed line and the $t$-amplitude by a solid line. The dashed line with a cross at one vertex represents the kinetic energy operator. Lines with arrows pointing upwards represent particles, lines with arrows pointing downwards represent holes, and circles are always hole lines.

the linear term with summation over one particle and one hole index. The coupled ladder equations are

$$
\begin{aligned}
0 & =\left\langle\mathbf{k}_{a} \mathbf{k}_{b}|v| \mathbf{k}_{i} \mathbf{k}_{j}\right\rangle \\
& +P\left(\mathbf{k}_{a} \mathbf{k}_{b}\right) \sum_{\mathbf{k}_{c}}\left\langle\mathbf{k}_{b}|f| \mathbf{k}_{c}\right\rangle\left\langle\mathbf{k}_{a} \mathbf{k}_{c}|t| \mathbf{k}_{i} \mathbf{k}_{j}\right\rangle \\
& -P\left(\mathbf{k}_{i} \mathbf{k}_{j}\right) \sum_{\mathbf{k}_{k}}\left\langle\mathbf{k}_{a} \mathbf{k}_{b}|t| \mathbf{k}_{i} \mathbf{k}_{k}\right\rangle\left\langle\mathbf{k}_{k}|f| \mathbf{k}_{j}\right\rangle \\
& +\frac{1}{2} \sum_{\mathbf{k}_{c} \mathbf{k}_{d}}\left\langle\mathbf{k}_{a} \mathbf{k}_{b}|v| \mathbf{k}_{c} \mathbf{k}_{d}\right\rangle\left\langle\mathbf{k}_{c} \mathbf{k}_{d}|t| \mathbf{k}_{i} \mathbf{k}_{j}\right\rangle \\
& +\frac{1}{2} \sum_{\mathbf{k}_{k} \mathbf{k}_{l}}\left\langle\mathbf{k}_{a} \mathbf{k}_{b}|t| \mathbf{k}_{k} \mathbf{k}_{l}\right\rangle\left\langle\mathbf{k}_{k} \mathbf{k}_{l}|v| \mathbf{k}_{i} \mathbf{k}_{j}\right\rangle
\end{aligned}
$$

We define the particle-particle ladder equations (PPLAD) as Eq. (8) where the last line, the hole-hole ladder diagram, is removed. In the PPHH-LAD approximation, the five first diagrams of Fig. 1 are retained. The PPLAD approximation uses only the four first diagrams in the same figure.

The coupled-cluster energy equation $(3)$ becomes in the CCD approximation

$$
E_{C C D}=E_{R E F}+\Delta E_{C C D},
$$

where the reference energy is written as

$$
E_{R E F}=\sum_{\mathbf{k}_{i}}\left\langle\mathbf{k}_{i}\left|h_{0}\right| \mathbf{k}_{i}\right\rangle+\frac{1}{2} \sum_{\mathbf{k}_{i} \mathbf{k}_{j}}\left\langle\mathbf{k}_{i} \mathbf{k}_{j}|v| \mathbf{k}_{i} \mathbf{k}_{j}\right\rangle,
$$

and the correlation correction term is simply

$$
\Delta E_{C C D}=\frac{1}{4} \sum_{\mathbf{k}_{a} \mathbf{k}_{b} \mathbf{k}_{i} \mathbf{k}_{j}}\left\langle\mathbf{k}_{i} \mathbf{k}_{j}|v| \mathbf{k}_{a} \mathbf{k}_{b}\right\rangle\left\langle\mathbf{k}_{a} \mathbf{k}_{b}|t| \mathbf{k}_{i} \mathbf{k}_{j}\right\rangle .
$$

The general expressions for the CC energy are derived in for example Ref. 33. A diagrammatic representation of the energy equation is given in Fig. 2. The correlation energy has more terms in a general case when the $\hat{T}_{1}$ amplitude does not vanish.

Let us define $T$ as the amplitude matrix, with the matrix elements

$$
[T]_{\alpha, \beta}=\left\langle\mathbf{k}_{p(\alpha)} \mathbf{k}_{q(\alpha)}|t| \mathbf{k}_{r(\beta)} \mathbf{k}_{s(\beta)}\right\rangle,
$$

where $p, q, r$, and $s$ are functions of the two-body configurations $\alpha$ and $\beta$. As explained in Ref. [33, the ladder 
ALGORITHM I. Fixed-point iteration scheme for solving the ladder equations, as explained in Ref. 33. The amplitude matrix $T$ and the function $z$ are defined in the text.

1. Initialize $E_{\text {old }}$ to a large number.

2. Initialize the amplitude matrix $T_{\text {old }}$ to zero.

3. Loop until convergence:

(a) Calculate $T_{\text {new }}=z\left(T_{\text {old }}\right)$.

(b) Calculate a new binding energy

$E_{\text {new }}=\Delta E_{C C D}\left(T_{\text {new }}\right)$.

If $\left|E_{\text {new }}-E_{\text {old }}\right|$ is smaller than a given tolerance, stop.

Else, set $E_{\text {old }}=E_{\text {new }}$ and $T_{\text {old }}=T_{\text {new }}$ and return to $3 \mathrm{a}$.

equations can be written as the more convenient matrix equation

$$
T=z(T)
$$

where the left hand side consists of only an amplitude matrix and the rest of the ladder equations, here written as the function $z$ of the amplitude matrix $T$, is on the right hand side. Utilizing the representation of Eq. (11), the amplitude equation can be solved by a fixed-point iteration scheme. Algorithm I is commonly used in $\mathrm{CC}$ calculations [33, and this is the procedure we have employed.

\section{B. Brueckner-Hartree-Fock approximation}

In Brueckner-Hartree-Fock (BHF) theory [12, 67, the total energy is approximated by

$$
E_{B H F}=\sum_{\mathbf{k}_{i}}\left\langle\mathbf{k}_{i}\left|h_{0}\right| \mathbf{k}_{i}\right\rangle+\frac{1}{2} \sum_{\mathbf{k}_{i} \mathbf{k}_{j}}\left\langle\mathbf{k}_{i} \mathbf{k}_{j}|g| \mathbf{k}_{i} \mathbf{k}_{j}\right\rangle,
$$

where the $G$-matrix is defined as

$$
\begin{aligned}
& \left\langle\mathbf{k}_{p} \mathbf{k}_{q}|g| \mathbf{k}_{r} \mathbf{k}_{s}\right\rangle=\left\langle\mathbf{k}_{p} \mathbf{k}_{q}|v| \mathbf{k}_{r} \mathbf{k}_{s}\right\rangle \\
& +\frac{1}{2} \sum_{\mathbf{k}_{c} \mathbf{k}_{d}} \frac{\left\langle\mathbf{k}_{p} \mathbf{k}_{q}|v| \mathbf{k}_{c} \mathbf{k}_{d}\right\rangle\left\langle\mathbf{k}_{c} \mathbf{k}_{d}|g| \mathbf{k}_{r} \mathbf{k}_{s}\right\rangle}{\varepsilon_{\mathbf{k}_{p}}+\varepsilon_{\mathbf{k}_{q}}-\varepsilon_{\mathbf{k}_{c}}-\varepsilon_{\mathbf{k}_{d}}} .
\end{aligned}
$$

and the single-particle energy is

$$
\varepsilon_{\mathbf{k}_{p}}=\left\langle\mathbf{k}_{p}\left|h_{0}\right| \mathbf{k}_{p}\right\rangle+\sum_{\mathbf{k}_{i}}\left\langle\mathbf{k}_{p} \mathbf{k}_{i}|g| \mathbf{k}_{p} \mathbf{k}_{i}\right\rangle .
$$

In the so-called continuous option 68, 69, which we use, the single-particle energy has the form given in Eq. 13 for both particle and hole states.

Diagrams of the BHF equations are given in Figs. 3 and 4. From the diagrammatic expressions one can see that the PP-LAD and BHF equations are similar. In fact, one can show that the BHF equations become equal

$$
E_{B H F}=0^{-x}+\text { Ommno }
$$

FIG. 3. The BHF energy equation in terms of diagrams. The waved line represents the $G$-matrix, and a circle means summation over hole states.

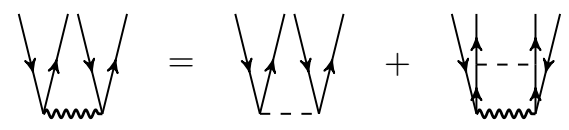

FIG. 4. Definition of the $G$-matrix, expressed using diagrams. The wavy line represents the $G$-matrix interaction. All other parts are defined as in Fig. 1

to the particle-particle ladder equations (PP-LAD), provided that the $G$-matrix in the single-particle energy expression 13 is replaced by the interaction matrix. We used this similarity to verify our implementation of the PP-LAD equations as well. In the PPHH-LAD approximation we have in addition to particle-particle ladders hole-hole ladder contributions, and the two types of ladder diagrams couple to each other.

\section{FURTHER APPROXIMATIONS}

Explicit expressions of the nuclear interaction are commonly given in relative momentum coordinates, whereas the coupled-cluster equations are defined in laboratory coordinates. Either the interaction may be transformed to laboratory coordinates, or the many-body equations must be rewritten to relative coordinates. We chose to transform the ladder equations from laboratory to relative and center-of-mass (RCM) coordinates. Details of the transformation are shown in App. A 1.

In addition to transforming the equations from laboratory to $\mathrm{RCM}$ coordinates, we write the equations in a basis of coupled angular momenta. The basis we use is $\left|k(l S) \mathcal{J} m_{\mathcal{J}} M_{T}\right\rangle$, where $k$ is the radial component of the relative momentum, $l$ is the relative orbital angular momentum, $S$ is the total two-particle spin, $\mathcal{J}$ is the angular momentum sum $l+S, m_{\mathcal{J}}$ is the $z$ projection of $\mathcal{J}$, and $M_{T}$ is the projection of the two-particle isospin. The same representation is commonly used in nuclear structure calculations, such as the Brueckner-HartreeFock method [12], but due to angular-average approximations the matrix elements are normally diagonal in $\mathcal{J}$ and $m_{\mathcal{J}}$.

As can be seen from Eq. A4, the ladder equations in RCM coordinates depend on the particle-particle Pauli exclusion operator

$Q_{p p}^{(e)}\left(\mathbf{k}, \mathbf{K}, k_{F}\right)=\theta\left(|\mathbf{k}+\mathbf{K} / 2|-k_{F}\right) \theta\left(|-\mathbf{k}+\mathbf{K} / 2|-k_{F}\right)$

and the hole-hole Pauli exclusion operator

$Q_{h h}^{(e)}\left(\mathbf{k}, \mathbf{K}, k_{F}\right)=\theta\left(k_{F}-|\mathbf{k}+\mathbf{K} / 2|\right) \theta\left(k_{F}-|-\mathbf{k}+\mathbf{K} / 2|\right)$,

where $k_{F}$ is the Fermi momentum, $\mathbf{k}$ and $\mathbf{K}$ are relative and CM momenta, respectively, defined in Eq. (A3), and 
the superscript (e) emphasizes that these are the exact Pauli operators. A common approximation for nuclear matter calculations is to replace the exact Pauli operators by an operator averaged over the angle between the relative and CM momentum vectors [12, 54, 67.

In this paper, we will use a technique introduced by Suzuki et al. [15] to expand the exact Pauli operator in partial waves. Using the exact Pauli operator in a partial wave expansion, we derive the CC ladder equations. We will also derive the ladder equations using angularaverage approximations of the Pauli operator. In both cases we will use an angular-average approximation of the single-particle energies.

\section{A. Exact Pauli operator}

Expressed in the coupled partial wave basis, the reference energy per particle is

$$
\begin{aligned}
E_{R E F} / A= & \frac{3 \hbar^{2} k_{F}^{2}}{10 m}+\frac{3 C}{4 k_{F}^{3}} \sum_{\mathcal{J} S l} \sum_{M_{T}}(2 \mathcal{J}+1) \\
& \times \int_{0}^{2 k_{F}} d K K^{2} \int_{0}^{\sqrt{k_{F}^{2}-K^{2} / 4}} d k k^{2} \\
& \times\left\langle k(l S) \mathcal{J} M_{T}|v| k(l S) \mathcal{J} M_{T}\right\rangle \\
& \times x_{h h}\left(k, K, k_{F}\right),
\end{aligned}
$$

where $A$ is the number of particles, $k_{F}$ is the Fermi momentum, $k$ and $K$ are the radial coordinates of the relative and $\mathrm{CM}$ momentum, respectively, and $C$ is 1 for symmetric nuclear matter and 2 for pure neutron matter. The function $x_{h h}$ is defined as

$x_{h h}= \begin{cases}0, & \text { if } k>\sqrt{k_{F}^{2}-K^{2} / 4}, \\ -\frac{k^{2}-k_{F}^{2}+K^{2} / 4}{k K}, & \text { if } k_{F}-K / 2<k<\sqrt{k_{F}^{2}-K^{2} / 4}, \\ 1, & \text { otherwise }\end{cases}$

and similarly we define a function

$x_{p p}= \begin{cases}0, & \text { if } k<\sqrt{k_{F}^{2}-K^{2} / 4}, \\ \frac{k^{2}-k_{F}^{2}+K^{2} / 4}{k K}, & \text { if } \sqrt{k_{F}^{2}-K^{2} / 4}<k<k_{F}+K / 2, \\ 1, & \text { otherwise. }\end{cases}$

In Sec. III, all interaction and $t$-amplitude matrix elements are assumed to be multiplied by the antisymmetrization factor $\mathcal{A}^{l^{\prime} l S M_{T}}$ given in Eq. A9. Since the Pauli exclusion operator is the only factor in the potential energy part of the reference energy that depends on the angle between $\mathbf{k}$ and $\mathbf{K}$, the expression for the reference energy is the same when using exact and angularaveraged Pauli operators. As mentioned above, the reference energy is also the Hartree-Fock energy when using the plane wave basis for this particular system. In the limit of an untruncated basis, the reference energy expressed in a partial wave basis, given in Eq. (14), equals

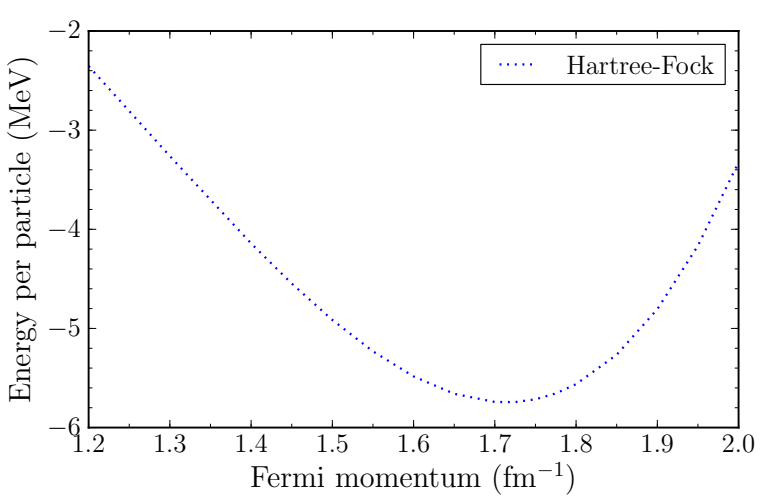

FIG. 5. (Color online) Total energy per nucleon of symmetric nuclear matter in the Hartree-Fock approximation, given as a function of Fermi momentum $k_{F}$. The calculation was done with a bare $\mathrm{N}^{3} \mathrm{LO}$ two-body interaction, and the total angular momentum truncation was set to $\mathcal{J} \leq 24$.

the Hartree-Fock energy. Since we calculate the reference energy with a high cutoff in angular momentum, we will refer to the reference energy (14) as the HartreeFock energy. However, one should notice that the partial wave basis is not a Hartree-Fock basis for infinite nuclear matter. The reference energy is plotted in Fig. 5.

In the algorithm used to solve the PP-LAD and PPHHLAD equations, it is necessary to store all $t$-amplitude matrix elements to be able to calculate the binding energy at each iteration step. When using a sufficient number of integration grid points and angular momentum partial waves to reach necessary accuracy, the size of the CC amplitude matrix would typically exceed standard memory capacities at most high-performance computing facilities. When the ladder equations are written in the coupled partial wave basis we used, it is possible to calculate the $t$-amplitude matrix for only one angular direction of the CM momentum $\mathbf{K}$, and then obtain the other matrix elements by performing a rotation [15. This is a major advantage, since the memory requirements for storing the $t$-amplitude decreases significantly.

The size of the $t$-amplitude matrix can be further decreased by removing the dependency on the angular parts of the relative momenta, that is, $\hat{\mathbf{k}}$ and $\hat{\mathbf{k}}^{\prime}$. As can be seen from Eq. A4, the only dependency on these vectors in the ladder amplitude equations that cannot be separated out occurs in the single-particle energies. In RCM coordinates, the single-particle energies are functions of $| \pm \mathbf{k}+\mathbf{K} / 2|$ or $\left| \pm \mathbf{k}^{\prime}+\mathbf{K} / 2\right|$, as shown in Eq. (A11). To remove the dependency of the $t$-amplitude matrix on $\hat{\mathbf{k}}$ and $\hat{\mathbf{k}}^{\prime}$, we use an angular-average approximation of the arguments $| \pm \mathbf{k}+\mathbf{K} / 2|$ in the single-particle energy [54, 67, 70. Since nuclear matter is an isotropic medium, the single-particle energy must be a symmetric function, and the single-particle energy can be approximated as a finite polynomial with only even powers. Following 
Ramos [54, 70, we replace the input momentum

$$
k_{p}=| \pm \mathbf{k}+\mathbf{K} / 2|,
$$

by the angular-average approximation

$$
\overline{k_{p}}=\sqrt{k^{2}+K^{2} / 4 \pm k K \sqrt{\left\langle\cos ^{2} \theta_{\mathbf{k K}}\right\rangle}},
$$

where

$$
\left\langle\cos ^{2} \theta_{\mathbf{k K}}\right\rangle= \begin{cases}x_{h h}^{3}(k, K) / 3, & \text { if } k_{p} \leq k_{F}, \\ x_{p p}^{3}(k, K) / 3, & \text { if } k_{p}>k_{F}\end{cases}
$$

and $\theta_{\mathbf{k K}}$ is the angle between the relative and CM momentum vectors.

If we use the above mentioned rotation of the $t$ amplitude matrix, given explicitly in Eq. (A18), and apply the angular-average approximation in Eq. (17), we can write the correlation energy per particle as

$$
\begin{aligned}
& \Delta E_{C C D} / A=\frac{3 C}{32 k_{F}^{3}} \sum_{\mathcal{J} m_{\mathcal{J}}} \sum_{\mathcal{J}^{\prime \prime} m_{\mathcal{J}^{\prime \prime}}} \sum_{\mathcal{J}^{\prime \prime \prime} m_{\mathcal{J}^{\prime \prime \prime}}} \sum_{m_{\mathcal{J}^{\prime}}} \sum_{S M_{T}} \sum_{l l^{\prime} l^{\prime \prime} l^{\prime \prime \prime}} \\
& \times \int_{0}^{2 k_{F}} K^{2} d K \int_{-1}^{1} d \cos \theta_{K} \\
& \times \int_{0}^{\sqrt{k_{F}^{2}-K^{2} / 4}} k^{2} d k \int_{\sqrt{k_{F}^{2}-K^{2} / 4}}^{\infty} k^{\prime 2} d k^{\prime} \\
& \times d_{m_{\mathcal{J}^{\prime \prime}} m_{\mathcal{J}^{\prime}}}^{\mathcal{J}^{\prime \prime}}\left(\theta_{K}\right) d_{m_{\mathcal{J}^{\prime \prime \prime}} m_{\mathcal{J}^{\prime}}}^{\mathcal{J}^{\prime \prime \prime}}\left(\theta_{K}\right) \\
& \times\left\langle k(l S) \mathcal{J} M_{T}|v| k^{\prime}\left(l^{\prime} S\right) \mathcal{J} M_{T}\right\rangle \\
& \times\left\langle k^{\prime}\left(l^{\prime \prime} S\right) \mathcal{J}^{\prime \prime} m_{\mathcal{J}^{\prime}} M_{T}|t(K)| k\left(l^{\prime \prime \prime} S\right) \mathcal{J}^{\prime \prime \prime} m_{\mathcal{J}^{\prime}} M_{T}\right\rangle \\
& \times Q_{h h}\left(l^{\prime \prime \prime} \mathcal{J}^{\prime \prime \prime} m_{\mathcal{J}^{\prime \prime \prime}}, l \mathcal{J} m_{\mathcal{J}} ; S M_{T} k K \theta_{K}\right) \\
& \times Q_{p p}\left(l^{\prime} \mathcal{J} m_{\mathcal{J}}, l^{\prime \prime} \mathcal{J}^{\prime \prime} m_{\mathcal{J}^{\prime \prime}} ; S M_{T} k^{\prime} K \theta_{K}\right),
\end{aligned}
$$

where the Wigner $D$-function has been defined through

$$
D_{M M^{\prime}}^{J}(\alpha, \beta, \gamma)=e^{-i M \alpha} d_{M M^{\prime}}^{J}(\beta) e^{-i M^{\prime} \gamma},
$$

and the function $d_{M M^{\prime}}^{J}(\beta)$ is given in Ref. [71. In Eq. (18), the $t$-amplitude is independent on the angles $\hat{\mathbf{k}}$ and $\hat{\mathbf{k}}^{\prime}$, and we have used the definitions

$$
\begin{aligned}
& Q_{h h}\left(l^{\prime \prime} \mathcal{J}^{\prime \prime} m_{\mathcal{J}^{\prime \prime}}, l^{\prime \prime \prime} \mathcal{J}_{m_{\mathcal{J}}} ; S M_{T} k K \theta_{K} \phi_{K}\right)= \\
& \mathcal{A}^{l^{\prime \prime} l^{\prime \prime \prime} S M_{T}} \frac{1}{2} \sum_{m_{l^{\prime \prime \prime}} m_{l^{\prime \prime}}} \sum_{M_{S}} \int d \hat{\mathbf{k}} Y_{l^{\prime \prime} m_{l^{\prime \prime}}}^{*}(\hat{\mathbf{k}}) Y_{l^{\prime \prime \prime} m_{l^{\prime \prime \prime}}}(\hat{\mathbf{k}}) \\
& \times\left\langle l^{\prime \prime \prime} m_{l^{\prime \prime \prime}} S M_{S} \mid \mathcal{J} m_{\mathcal{J}}\right\rangle\left\langle l^{\prime \prime} m_{l^{\prime \prime}} S M_{S} \mid \mathcal{J}^{\prime \prime} m_{\mathcal{J}^{\prime \prime}}\right\rangle \\
& \times \theta\left(k_{F}-|\mathbf{k}+\mathbf{K} / 2|\right) \theta\left(k_{F}-|-\mathbf{k}+\mathbf{K} / 2|\right)
\end{aligned}
$$

and

$$
\begin{aligned}
& Q_{p p}\left(l^{\prime \prime} \mathcal{J}^{\prime} m_{\mathcal{J}^{\prime}}, l^{\prime \prime \prime} \mathcal{J}^{\prime \prime} m_{\mathcal{J}^{\prime \prime}} ; S M_{T} k^{\prime} K \theta_{K} \phi_{K}\right)= \\
& \mathcal{A}^{l^{\prime \prime} l^{\prime \prime \prime} S M_{T}} \frac{1}{2} \sum_{m_{l^{\prime \prime}} m_{l^{\prime \prime \prime}}} \sum_{M_{S}^{\prime}} \int d \hat{\mathbf{k}}^{\prime} Y_{l^{\prime \prime}}^{*} m_{l^{\prime \prime}}\left(\hat{\mathbf{k}}^{\prime}\right) Y_{l^{\prime \prime \prime} m_{l^{\prime \prime \prime}}}\left(\hat{\mathbf{k}}^{\prime}\right) \\
& \times\left\langle l^{\prime \prime} m_{l^{\prime \prime}} S M_{S}^{\prime} \mid \mathcal{J}^{\prime} m_{\mathcal{J}^{\prime}}\right\rangle\left\langle l^{\prime \prime \prime} m_{l^{\prime \prime \prime}} S M_{S}^{\prime} \mid \mathcal{J}^{\prime \prime} m_{\mathcal{J}^{\prime \prime}}\right\rangle \\
& \times \theta\left(\left|\mathbf{k}^{\prime}+\mathbf{K} / 2\right|-k_{F}\right) \theta\left(\left|-\mathbf{k}^{\prime}+\mathbf{K} / 2\right|-k_{F}\right)
\end{aligned}
$$

in a similar way as Suzuki et al. did in their BHF study 15. In Eqs. 20 and (21), the brackets denote ClebschGordan coefficients and the functions $Y_{l m_{l}}(\hat{\mathbf{k}})$ are spherical harmonics 71 .

In Ref. 15, the authors have derived an expression for $Q_{p p}$ which avoids the complicated integration limits over the space angle of $\mathbf{k}$ [15], namely

$$
\begin{aligned}
& Q_{p p}\left(l, \mathcal{J}, m_{\mathcal{J}}, l^{\prime} \mathcal{J}^{\prime} m_{\mathcal{J}^{\prime}} ; S M_{T} k K \theta_{K} \phi_{K}\right)= \\
& \mathcal{A}^{l l^{\prime} S M_{T}} \frac{1}{2}\left[x_{p p} \delta_{l l^{\prime}} \delta_{\mathcal{J} \mathcal{J}^{\prime}} \delta_{m_{\mathcal{J}} m_{\mathcal{J}^{\prime}}}\right. \\
& +\sum_{L>0 ; L=\text { even }}(-1)^{S+m_{\mathcal{J}}} \\
& \times \frac{(4 \pi)^{1 / 2} \hat{l} \hat{l^{\prime}} \hat{\mathcal{J}} \hat{\mathcal{J}}^{\prime}}{\hat{L}}\left\langle l 0 l^{\prime} 0 \mid L 0\right\rangle\left\langle\mathcal{J}-m_{\mathcal{J}} \mathcal{J}^{\prime} m_{\mathcal{J}^{\prime}} \mid L M_{L}\right\rangle \\
& \times Y_{L M_{L}}\left(\theta_{K}, \phi_{K}\right) W\left(l \mathcal{J} l^{\prime} \mathcal{J}^{\prime} ; S L\right) \\
& \times\left[P_{L+1}\left(x_{p p}\right)-P_{L-1}\left(x_{p p}\right)\right]
\end{aligned}
$$

where $\hat{x} \equiv \sqrt{2 x+1}, P_{L}(x)$ is the Legendre polynomial, $W\left(l \mathcal{J} l^{\prime} \mathcal{J}^{\prime} ; S L\right)$ denotes the Racah coefficient [71, and $x_{p p}$ is as defined in Eq. 16. We have used Eq. 22 to evaluate $Q_{p p}$ and a similar expression for evaluating $Q_{h h}$. The expression for $Q_{h h}$ was obtained by replacing $x_{p p}$ by the equivalent for hole-hole states, i.e. $x_{h h}$ given in Eq. (15). Observe that the simplified but exact Pauli operator expression (22) and the corresponding expression for the hole-hole Pauli operator could not have been used if the $t$-amplitude was not independent on the angular vectors $\hat{\mathbf{k}}$ and $\hat{\mathbf{k}}^{\prime}$. The reason for this restriction is that the Pauli operator expressions, when expanded in partial waves, as defined in Eqs. 20 and (21), are integral operators and not just real functions. The effect of the integral operators $Q_{h h}$ and $Q_{p p}$ can be seen clearly from the correlation energy expression in Eq. (A17). The angular-average approximation of the single-particle energies therefore simplifies the correlation energy expression significantly.

Since the exact Pauli operator is not diagonal in $\mathcal{J}$ and $m_{\mathcal{J}}$, both the $G$-matrix [15] and the $t$-amplitude matrix are not diagonal in the total angular momentum $\mathcal{J}$ and its projection $m_{\mathcal{J}}$. The fact that the amplitude matrix is not diagonal in total angular momentum makes the ladder equations more complicated. Writing the PPHH- 
LAD equations in the basis $\left|k(l S) \mathcal{J} m_{\mathcal{J}} M_{T}\right\rangle$, we get

$$
\begin{aligned}
& \Delta \tilde{\varepsilon}\left(k, k^{\prime}, K\right)\left\langle k^{\prime}\left(l^{\prime} S\right) \mathcal{J}^{\prime} m_{\mathcal{J}^{\prime}} M_{T}|t(K)| k(l S) \mathcal{J}_{m_{\mathcal{J}}} M_{T}\right\rangle \\
& =\left\langle k^{\prime}\left(l^{\prime} S\right) \mathcal{J}^{\prime} m_{\mathcal{J}^{\prime}} M_{T}|v| k(l S) \mathcal{J} m_{\mathcal{J}} M_{T}\right\rangle \delta_{\mathcal{J}} \delta_{\mathcal{J}^{\prime}} \delta_{m_{\mathcal{J}} m_{\mathcal{J}^{\prime}}} \\
& +\frac{1}{2} \sum_{\mathcal{J}^{\prime \prime} m_{\mathcal{J}^{\prime \prime}}} \sum_{l^{\prime \prime} l^{\prime \prime \prime}} \int_{0}^{k_{F}} h^{2} d h \\
& \times\left\langle k^{\prime}\left(l^{\prime} S\right) \mathcal{J}^{\prime} m_{\mathcal{J}^{\prime}} M_{T}|t(K)| h\left(l^{\prime \prime} S\right) \mathcal{J}^{\prime \prime} m_{\mathcal{J}^{\prime \prime}} M_{T}\right\rangle \\
& \times\left\langle h\left(l^{\prime \prime \prime} S\right) \mathcal{J}_{\mathcal{J}^{\prime}} M_{T}|v| k(l S) \mathcal{J} m_{\mathcal{J}} M_{T}\right\rangle \\
& \times Q_{h h}\left(l^{\prime \prime} \mathcal{J}^{\prime \prime} m_{\mathcal{J}^{\prime \prime}}, l^{\prime \prime \prime} \mathcal{J} m_{\mathcal{J}} ; S M_{T} h K \theta_{K} \phi_{K}\right) \\
& +\frac{1}{2} \sum_{\mathcal{J}^{\prime \prime} m_{\mathcal{J}^{\prime \prime}} \sum_{l^{\prime \prime} l^{\prime \prime \prime}} \int_{0}^{\infty} p^{2} d p} \times\left\langle k^{\prime}\left(l^{\prime} S\right) \mathcal{J}^{\prime} m_{\mathcal{J}^{\prime}} M_{T}|v| p\left(l^{\prime \prime} S\right) \mathcal{J}^{\prime} m_{\mathcal{J}^{\prime}} M_{T}\right\rangle \\
& \times\left\langle p\left(l^{\prime \prime \prime} S\right) \mathcal{J}^{\prime \prime} m_{\mathcal{J}^{\prime \prime}} M_{T}|t(K)| k(l S) \mathcal{J} m_{\mathcal{J}} M_{T}\right\rangle \\
& \times Q_{p p}\left(l^{\prime \prime} \mathcal{J}^{\prime} m_{\mathcal{J}^{\prime}}, l^{\prime \prime \prime} \mathcal{J}^{\prime \prime} m_{\mathcal{J}^{\prime \prime}} ; S M_{T} p K \theta_{K} \phi_{K}\right),
\end{aligned}
$$

where $\left|(l S) \mathcal{J} m_{\mathcal{J}}\right\rangle$ denotes a vector where $l$ and $S$ are coupled to $\mathcal{J}$. In Eq. (23) we have used the angularaveraged energy denominator $\Delta \tilde{\varepsilon}\left(k, k^{\prime}, K\right)$, which is defined in Eq. A16). When using the rotation of the ladder amplitude matrix, given in Eq. A18, the amplitude matrix needs to be evaluated only at a single angular coordinate of the CM momentum. The amplitude equation (23) is therefore given as a function of only the radial part of the CM momentum.

In Eq. 22, the restriction that $L$ must be even ensures that parity is conserved [15]. This follows from the properties of the first Clebsch-Gordan coefficient in Eq. 22), which vanishes when $(-1)^{l} \neq(-1)^{l^{\prime}}$, provided that $L$ is even. Since the operators $Q_{h h}$ and $Q_{p p}$ conserve parity, one can see from Eq. (23) that the $t$-amplitude of the ladder equation also conserves parity. On the contrary, the exact Pauli operators (both particle-particle and hole-hole ones) do not conserve the total angular momentum $\mathcal{J}$. Consequently, the $t$-amplitude also becomes non-diagonal in the total angular momentum. The exact Pauli operators become diagonal in the projection $m_{\mathcal{J}}$ in the special case when the angular part of the CM momentum is zero. The $t$-amplitude matrix elements are therefore also diagonal in $m_{\mathcal{J}}$ when $\mathbf{K}$ is parallel with the $z$-axis.

We will later refer to the approximation with exact Pauli operators (both particle-particle and hole-hole ones) and angular-averaged single-particle energies as 'exact'. The angular-average approximation of the singleparticle energies, given in Eq. (17), is used in all calculations presented in this work, including both the coupled ladder approximations and the Brueckner-Hartree-Fock method.

\section{B. Angular-averaged Pauli operators}

The ladder equations can be simplified substantially by doing an angular-average approximation of the Pauli exclusion operators. The hole-hole and particle-particle exclusion operators become [15], respectively,

$$
\begin{aligned}
& Q_{h h}\left(l \mathcal{J} m_{\mathcal{J}}, l^{\prime} \mathcal{J}^{\prime} m_{\mathcal{J}^{\prime}} ; S M_{T} h K \theta_{K} \phi_{K}\right) \\
& \rightarrow \bar{Q}_{h h} \equiv \frac{1}{4 \pi} \int d \hat{\mathbf{K}} Q_{h h}\left(l \mathcal{J} m_{\mathcal{J}}, l^{\prime} \mathcal{J}^{\prime} m_{\mathcal{J}^{\prime}} ; S M_{T} h K \theta_{K} \phi_{K}\right) \\
& =\mathcal{A}^{l l S M_{T}} \frac{1}{2} x_{h h} \delta_{l l^{\prime}} \delta_{\mathcal{J} \mathcal{J}^{\prime}} \delta_{m_{\mathcal{J}} m_{\mathcal{J}^{\prime}}}
\end{aligned}
$$

and

$$
\begin{aligned}
& Q_{p p}\left(l \mathcal{J} m_{\mathcal{J}}, l^{\prime} \mathcal{J}^{\prime} m_{\mathcal{J}^{\prime}} ; S M_{T} p K \theta_{K} \phi_{K}\right) \\
& \rightarrow \bar{Q}_{p p} \equiv \frac{1}{4 \pi} \int d \hat{\mathbf{K}} Q_{p p}\left(l \mathcal{J}_{m_{\mathcal{J}}}, l^{\prime} \mathcal{J}^{\prime} m_{\mathcal{J}^{\prime}} ; S M_{T} p K \theta_{K} \phi_{K}\right) \\
& =\mathcal{A}^{l l S M_{T}} \frac{1}{2} x_{p p} \delta_{l l^{\prime}} \delta_{\mathcal{J} \mathcal{J}^{\prime}} \delta_{m_{\mathcal{J}} m_{\mathcal{J}^{\prime}}},
\end{aligned}
$$

where $x_{h h}$ and $x_{p p}$ are as defined in Eqs. (15) and (16). We note that the angular-averaged Pauli exclusion operators are diagonal in the total angular momentum $\mathcal{J}$, in contrast to the exact operators.

When using the angular-average approximation, the PPHH-LAD equations simplify to

$$
\begin{aligned}
& \Delta \tilde{\varepsilon}\left(k, k^{\prime}, K\right)\left\langle k^{\prime}\left(l^{\prime} S\right) \mathcal{J} M_{T}|t(K)| k(l S) \mathcal{J} M_{T}\right\rangle \\
& =\left\langle k^{\prime}\left(l^{\prime} S\right) \mathcal{J} M_{T}|v| k(l S) \mathcal{J} M_{T}\right\rangle \\
& +\frac{1}{2} \sum_{l^{\prime \prime}} \int_{0}^{k_{F}} h^{2} d h\left\langle k^{\prime}\left(l^{\prime} S\right) \mathcal{J} M_{T}|t(K)| h\left(l^{\prime \prime} S\right) \mathcal{J} M_{T}\right\rangle \\
& \times\left\langle h\left(l^{\prime \prime} S\right) \mathcal{J} M_{T}|v| k(l S) \mathcal{J} M_{T}\right\rangle \bar{Q}_{h h}(h, K) \\
& +\frac{1}{2} \sum_{l^{\prime \prime}} \int_{0}^{\infty} p^{2} d p\left\langle k^{\prime}\left(l^{\prime} S\right) \mathcal{J} M_{T}|v| p\left(l^{\prime \prime} S\right) \mathcal{J} M_{T}\right\rangle \\
& \times\left\langle p\left(l^{\prime \prime} S\right) \mathcal{J} M_{T}|t(K)| k(l S) \mathcal{J} M_{T}\right\rangle \bar{Q}_{p p}(p, K),
\end{aligned}
$$

where $\Delta \tilde{\varepsilon}\left(k, k^{\prime}, K\right)$ is the energy denominator with angular-averaged arguments. From the properties of the angular-averaged Pauli operators, it follows that the $t$ amplitude is diagonal in $\mathcal{J}$, and independent on $m_{\mathcal{J}}$ and the CM momentum angles $\theta_{K}$ and $\phi_{K}$. Because of these symmetries, the CC amplitude matrix is orders of magnitude smaller in the angular-averaged approximation than when using exact Pauli exclusion operators.

The CCD correlation energy per particle becomes in the angular-averaged approximation

$$
\begin{aligned}
\Delta E_{C C D}^{a v e} / A & =\frac{3 C}{16 k_{F}^{3}} \sum_{\mathcal{J}} \sum_{S M_{T}} \sum_{l l^{\prime}}(2 \mathcal{J}+1) \\
& \times \int_{0}^{\sqrt{k_{F}^{2}-K^{2} / 4}} k^{2} d k \int_{\sqrt{k_{F}^{2}-K^{2} / 4}}^{\infty} k^{\prime 2} d k^{\prime} \\
& \times \int_{0}^{2 k_{F}} K^{2} d K\left\langle k(l S) \mathcal{J} M_{T}|v| k^{\prime}\left(l^{\prime} S\right) \mathcal{J} M_{T}\right\rangle \\
& \times\left\langle k^{\prime}\left(l^{\prime} S\right) \mathcal{J} M_{T}|t(K)| k(l S) \mathcal{J} M_{T}\right\rangle \\
& \times \bar{Q}_{h h}(k, K) \bar{Q}_{p p}\left(k^{\prime}, K\right) .
\end{aligned}
$$


The approximation using both angular-averaged Pauli operators and angular-averaged arguments in the singleparticle energies will in the following be referred to as 'average'.

\section{RESULTS AND DISCUSSION}

In the following we will present results of numerical calculations using the above mentioned ladder approximations. These approximations are compared with conventional Brueckner-Hartree-Fock theory. We investigate also the role of angular-averaged Pauli exclusion operators and compare this with the exact treatment discussed above. In addition, we compare results obtained using the optimized $\mathrm{NNLO}_{\text {opt }}$ two-body interaction 63 with calculations done with the $\mathrm{N}^{3} \mathrm{LO}$ interaction 64. The different interaction models and many-body methods are applied to both symmetric nuclear matter and neutron matter systems.

In all our calculations, we have taken into account charge symmetry breaking and charge independence breaking of the chiral interactions. The BHF calculations were done using continuous single-particle energies 72, which here means that single-particle energies for both particles and holes were calculated using Eq. 13. The singularities in the $G$-matrix equation due to the continuous single-particle energies were avoided by calculating the principal value of the integral in the $G$-matrix equation. The $G$-matrix equation $\sqrt{12}$ was solved in a coupled angular momentum basis $\left|k(l S) \mathcal{J} m_{\mathcal{J}} M_{T}\right\rangle$ using the matrix inversion method of Haftel and Tabakin [12]. Unless stated explicitly otherwise, the BHF calculations have been calculated with a truncation of the total angular momentum at $\mathcal{J} \leq 24$ in the Born approximation and $\mathcal{J} \leq 9$ for the full $G$-matrix. The BHF calculations were done with angular-averaged Pauli operators, as described in Ref. 12.

The coupled ladder equations were solved both with exact and angular-averaged Pauli exclusion operators. We refer to these two approximations as 'exact' and 'average', respectively. All calculations were done with an angular-average approximation of the single-particle energies, as was explained in Sec. III A. The Hartree-Fock energy was calculated with a cutoff in total angular momentum at $\mathcal{J} \leq 24$. The correlation energy was calculated with a trunction at $\mathcal{J} \leq 16$ in the 'average' approximation and $\mathcal{J} \leq 8$ in the 'exact' approximation. Fig. 6 shows the convergence of the correlation energy as a function of the total angular momentum cutoff $\mathcal{J}_{\text {max }}$. Because of the high density, the angular momentum barrier cannot keep particles far apart from each other, and therefore the convergence as a function of total angular momentum is slow in infinite nuclear matter. At high angular momenta, the interaction is dominated by the one-pion exchange part, and the convergence behavior as a function of total angular momentum is therefore similar for different interaction models. Due to restrictions

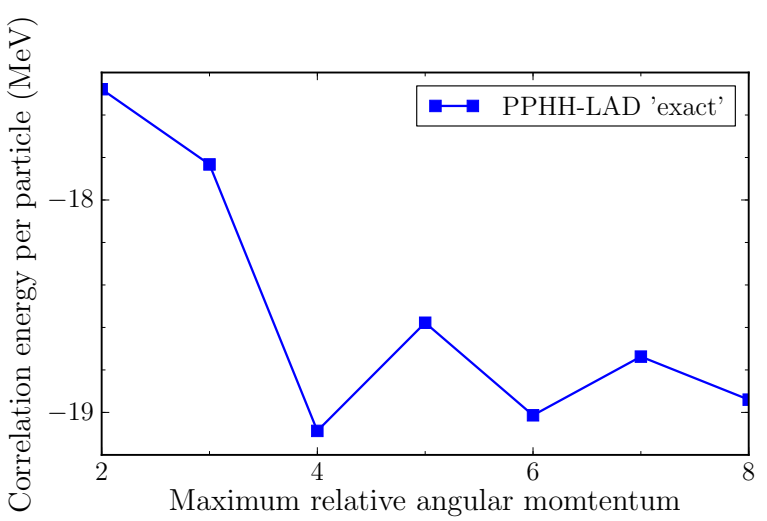

FIG. 6. (Color online) Convergence of the correlation energy of symmetric nuclear matter, given as a function of maximum total angular momentum $\mathcal{J}_{\max }$. The correlation energy was calculated for the Fermi momentum $k_{F}=1.8 \mathrm{fm}^{-1}$, using the PPHH-LAD approximation and exact Pauli exclusion operators.

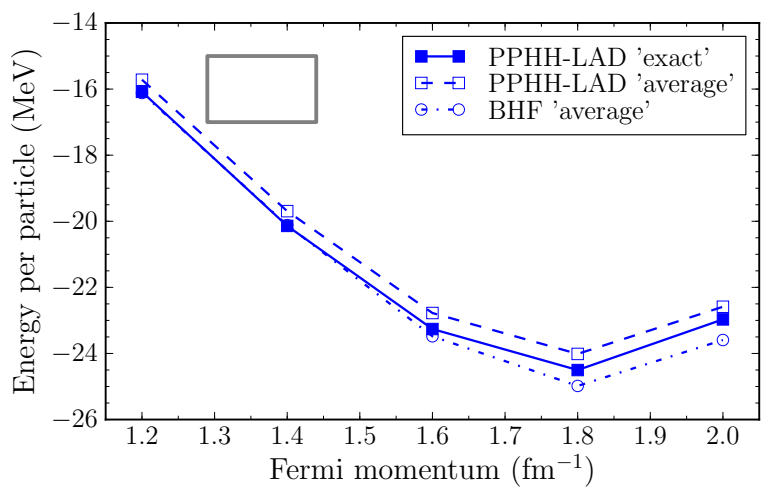

FIG. 7. (Color online) Total energy per particle as a function of the Fermi momentum, calculated for symmetric nuclear matter. The two approximations of the PPHH-LAD equations, 'average' and 'exact', are compared with a BruecknerHartree-Fock calculation with angular-averaged Pauli exclusion operator. These calculations were done with the $\mathrm{N}^{3} \mathrm{LO}$ 64 two-body interaction. The box denotes the uncertainty region for the experimental saturation point of symmetric nuclear matter, as obtained by extrapolating from observables of finite nuclei.

in computer memory, we were not able to calculate with $\mathcal{J}_{\text {max }}$ higher than 8 in the 'exact' approximation.

Let us first consider symmetric nuclear matter using the $\mathrm{N}^{3} \mathrm{LO}$ interaction. In Fig. 7, we compare the energy per nucleon as a function of the Fermi momentum for different approximations. The Fermi momentum at saturation is equal for all the three methods, whereas there are differences in the binding energies. The general form of the equation of state is very similar for the coupled lad- 
TABLE I. Total energy per nucleon at selected Fermi momenta $k_{F}$, as obtained with different approximations. For easier comparison, all these energies were calculated with the same cutoff in total angular momentum, i.e. $\mathcal{J}_{\max }=24$ for the Hartree-Fock / Born approximation and $\mathcal{J}_{\max }=8$ for the correlation contribution. All results were obtained with the $\mathrm{N}^{3} \mathrm{LO}$ interaction 64. Energies in units of $\mathrm{MeV}$.

\begin{tabular}{lccccc}
\hline \hline$k_{F}\left(\mathrm{fm}^{-1}\right)$ & 1.2 & 1.4 & 1.6 & 1.8 & 2.0 \\
\hline PPHH 'exact' & -16.08 & -20.14 & -23.26 & -24.50 & -22.97 \\
PPHH 'average' & -15.74 & -19.74 & -22.84 & -24.09 & -22.56 \\
PP 'exact' & -15.76 & -19.83 & -22.98 & -24.27 & -22.82 \\
PP 'average' & -15.45 & -19.45 & -22.57 & -23.86 & -22.40 \\
PT2 'average' & -15.11 & -19.81 & -23.35 & -24.80 & -23.25 \\
BHF 'average' & -16.18 & -20.25 & -23.74 & -25.47 & -24.42 \\
\hline \hline
\end{tabular}

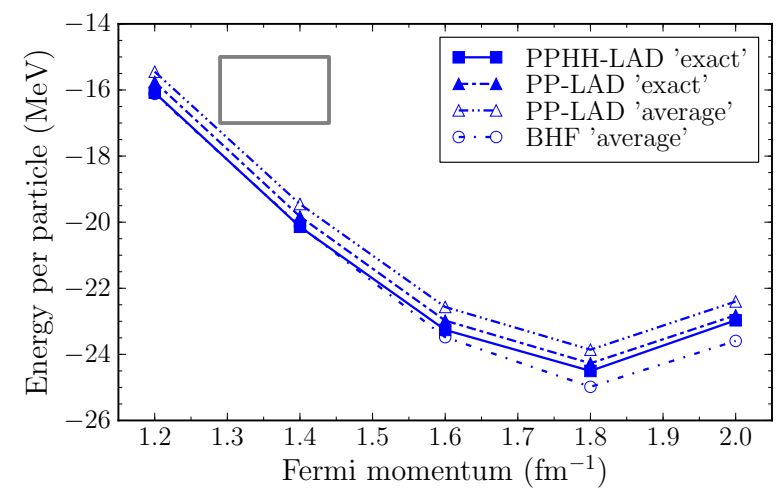

FIG. 8. (Color online) The 'exact' PPHH-LAD and PP-LAD calculations of energy per nucleon as a function of Fermi momentum. The equation of state of a Brueckner-Hartree-Fock calculation is also given. The box denotes the uncertainty region for the experimental saturation point of symmetric nuclear matter. All results were obtained with the $\mathrm{N}^{3} \mathrm{LO}$ interaction 64.

der and BHF methods. As can be seen from the figure, the PPHH-LAD approximation gives less binding than the BHF approximation both when the PPHH-LAD calculation is done with angular-averaged and exact Pauli operators. The binding energy at saturation obtained with the BHF method is approximately $0.5 \mathrm{MeV}$ lower than the corresponding result of Li et al. [6]. There are several factors that may have contributed to the difference between our BHF results and those of Li et al $[\underline{6}$. For example, in Ref. 6] they used a complex $G$-matrix, whereas we have used a real $G$-matrix and treated the singularities by using a principal value integral [12. It is also possible that we have used different angular-average approximations in the single-particle energies. As seen from Fig. 7, an exact treatment of the Pauli operators gives more binding than when using an angular-average approximation. This is in agreement with the results of Suzuki et al. [15] and Schiller et al. [14, where an ex-

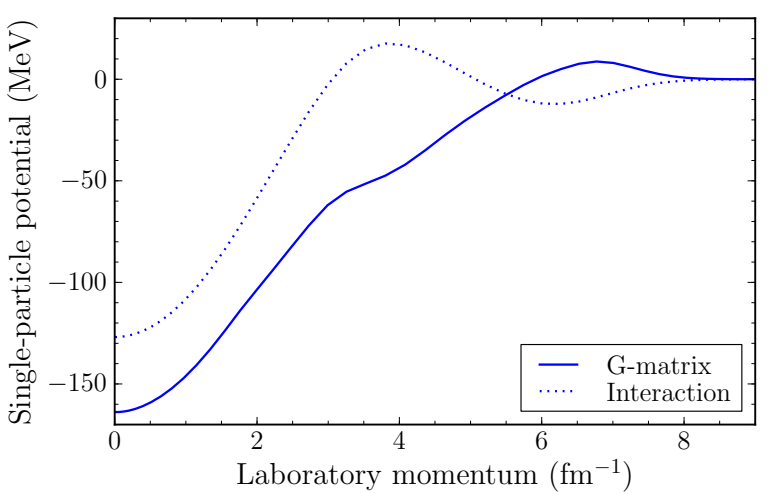

FIG. 9. (Color online) Single-particle potential as a function of laboratory frame momentum, calculated at Fermi momentum $1.8 \mathrm{fm}^{-1}$ with a $G$-matrix and with a bare interaction. In both cases, we used the $\mathrm{N}^{3} \mathrm{LO}$ two-body interaction and the total angular momentum cutoff $\mathcal{J}_{\max }=9$. The only difference between the Brueckner-Hartree-Fock and PP-LAD equations is the single-particle energy: In BHF theory the single-particle potential is calculated using a self-consistent $G$-matrix, whereas in the PP-LAD approximation the singleparticle energy is obtained by replacing the $G$-matrix with a bare interaction.

act treatment of the Pauli operator gave approximately $0.2-0.5 \mathrm{MeV}$ more binding energy in the BHF approximation.

Carbone et al. 8, have compared correlation energies obtained with the the self-consistent Green's function (SCGF) method at finite temperature with BHF calculations, using the same $\mathrm{N}^{3} \mathrm{LO}$ two-body interaction as we have used. Similarly as in Fig. 7, they got slightly higher energies with the SCGF method, which contains both particle-particle and hole-hole ladders, compared to the BHF results. In previous studies where the SCGF method has been compared with the BHF approximation [16, 55, 56 using other two-body interactions, the saturation energies obtained using the SCGF method have been located several $\mathrm{MeVs}$ higher than the corresponding BHF result, and the saturation densities have been shifted towards lower values. As will be shown systematically in a future publication 73 , we find a similar difference between the PPHH-LAD and BHF methods when using the hard-core Argonne $v_{18}$ interaction [74]. In fact, when using the Argonne $v_{18}$ potential, the saturation energy of the PPHH-LAD approximation is found to be only about $1 \mathrm{MeV}$ below the SCGF saturation energy shown in Fig. 3 of Ref. [16], and the saturation density is almost the same for both ladder approximations. It is interesting to note that we observed a larger difference between our $\mathrm{CC}$ and BHF results in systems with a hard interaction compared to systems with a soft interaction. If we relate these findings with a hard-core interaction to those obtained with for example the Bochum CC truncation scheme 44 mentioned in the introduction, it may be 
possible that the Bochum scheme will give a faster convergence than the $\mathrm{SUB} n$ truncation scheme when using hard-core interaction models.

Finally, we ought to mention that the way the CC equations are solved here, and in most other CC applications as well, no self-consistent solution of the pairing gap equations is performed. In practical terms this means that we never face instabilities in the denominators of the CC expansions due to poles arising in the two-hole sector. In CC theory, in contrast to various SCGF approaches, there is never an explicit energy dependence in the denominators of the different amplitudes. The energy differences in the two-particle-two-hole energies that enter the computation of various denominators are never zero, by construction. There are no terms in our present formalism which thus could account for pairing instabilities in the denominators, as discussed in for example Refs. [75, 76]. The effects of pairing instabilities and the self-consistent solution of the gap equation together with the full CCD equations (including the particle-hole terms as well), await therefore further investigations.

In Table I. we list the total energies for symmetric nuclear matter calculated with the $\mathrm{N}^{3} \mathrm{LO}$ two-body interaction. For easier comparison, all results are computed with the same cutoffs in total angular momentum. We find that the difference between the PPHH-LAD energies with angular-averaged and exact Pauli operators is approximately $0.4 \mathrm{MeV}$ at the saturation Fermi momentum. This makes a difference of roughly $1.7 \%$. At the same Fermi momentum, the PPHH-LAD method with exact Pauli operators gives approximately $1 \mathrm{MeV}$ more binding than the BHF method with angular-averaged Pauli operators. The $1 \mathrm{MeV}$ difference corresponds to $3.8 \%$ of the total energy.

In Fig. 8 we compare the coupled particle-particle and hole-hole ladder approximation, PPHH-LAD, with the particle-particle ladder approximation, PP-LAD. From Fig. 8 one can see that the inclusion of hole-hole ladders gives slightly more binding compared to the pure particleparticle ladder approximation. From Table \we find that the difference is approximately $0.2 \mathrm{MeV}$ at saturation Fermi momentum, or about $1 \%$ of the binding energy. At the saturation density, the contribution coming from including the hole-hole ladders is smaller than the error of an angular-average approximation of the Pauli exclusion operators.

As mentioned earlier, the only difference between the BHF and PP-LAD approximations is the single-particle energy, which in the BHF method is calculated with a $G$-matrix and in the ladder approximation with a bare interaction. Single-particle potentials with a $G$-matrix and with a bare interaction are plotted in Fig. 9, as obtained using the $\mathrm{N}^{3} \mathrm{LO}$ interaction.

Next we compare the two different chiral interactions $\mathrm{N}^{3} \mathrm{LO}$ and $\mathrm{NNLO}_{\text {opt }}$ when applied to infinite matter systems. In Fig. 10 we have plotted the equations of state for symmetric nuclear matter and in Fig. 11 for pure neutron matter, respectively, as obtained with the two different

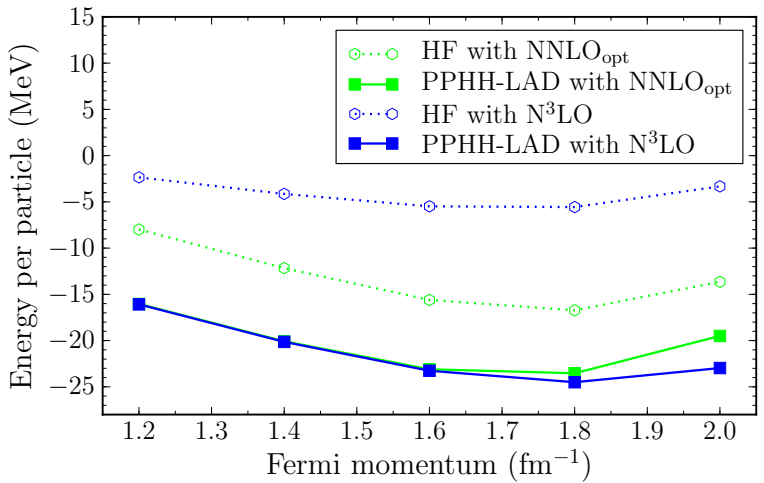

FIG. 10. (Color online) Energy per particle for symmetric nuclear matter, as calculated in the HF and PPHH-LAD approximations using the $\mathrm{N}^{3} \mathrm{LO}$ and $\mathrm{NNLO}_{\text {opt }}$ two-body interactions. In the PPHH-LAD approximation, the angular momentum cutoff was set to $\mathcal{J} \leq 8$ and the calculations were done with exact Pauli exclusion operators.

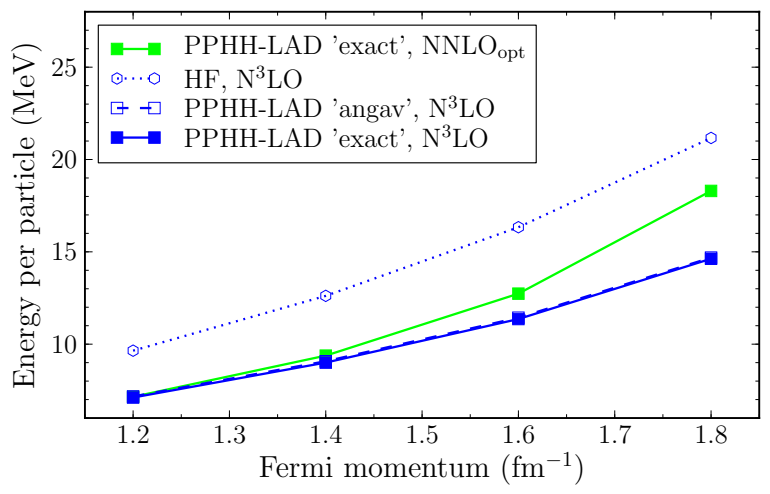

FIG. 11. (Color online) Energy per particle for pure neutron matter given as a function of Fermi momentum $k_{F}$. The figure shows results of calculations in Hartree-Fock (HF) and coupled particle-particle and hole-hole ladder approximation (PPHH-LAD), using the $\mathrm{N}^{3} \mathrm{LO}$ and $\mathrm{NNLO}_{\text {opt }}$ two-body interactions. The neutron matter results obtained with exact Pauli operators have been published in Ref. 63.

nuclear interaction models. When using the $\mathrm{NNLO}_{\mathrm{opt}}$ interaction for symmetric nuclear matter, we find that the HF energy is much closer to the PPHH-LAD approximation than is the case with the $\mathrm{N}^{3} \mathrm{LO}$ interaction. Thus, the optimized next-to-next-to-lowest-order interaction provides a better starting point for the perturbation series than the $\mathrm{N}^{3} \mathrm{LO}$ interaction. As can be seen from Fig. 10, in the PPHH-LAD approximation the two different interactions give almost the same equation of state for Fermi momenta less than $1.6 \mathrm{fm}^{-1}$. At higher Fermi momenta, the $\mathrm{NNLO}_{\mathrm{opt}}$ interaction gives less binding. Both the $\mathrm{N}^{3} \mathrm{LO}$ and $\mathrm{NNLO}_{\text {opt }}$ interactions overbinds considerably and saturates at too high density in symmet- 
ric nuclear matter. The similarity between the binding energies obtained with the two different two-body interactions is in contrast to the results in finite nuclei 63, where the $\mathrm{NNLO}_{\text {opt }}$ interaction gave significantly better agreement with experiments than the $\mathrm{N}^{3} \mathrm{LO}$ interaction. However, even if this may indicate that three-body forces could play a smaller role with the optimized interaction, there is no clear indication that such correlations are negligible. The $\mathrm{N}^{3} \mathrm{LO}$ and $\mathrm{NNLO}_{\text {opt }}$ interactions have also been compared in nuclear matter calculations using the SCGF method at finite temperature 8. The SCGF method was found to give slightly more binding when using the $\mathrm{NNLO}_{\text {opt }}$ interaction compared to calculations with the $\mathrm{N}^{3} \mathrm{LO}$ two-body interaction. The results obtained by Carbone et al. 8], using the SCGF method, are rather close to those obtained with our present CCD calculations.

Equations of state for neutron matter are given in Fig. 11. As can be seen from the figure, the differences between the calculations with exact and angular-averaged Pauli operators are much smaller for neutron matter than for symmetric nuclear matter. According to these results, the angular-average approximation of the Pauli operators is a fairly good approximation in neutron matter systems. In Ref. 63] we found that the equation of state for neutron matter with the optimized NNLO interaction was within the error estimates obtained with an $\mathrm{N}^{3} \mathrm{LO}$ interaction with three-body forces 24, whereas a calculation with a two-body $\mathrm{N}^{3} \mathrm{LO}$ interaction gave an equation of state that was more attractive around the empirical saturation density. Below we will show that the stronger repulsion seen when using the optimized $\mathrm{NNLO}_{\text {opt }}$ interaction stems from a poorer reproduction of the ${ }^{3} P_{0}$ and ${ }^{3} P_{1}$ partial wave phase shifts of the Nijmegen analysis.

The results for symmetric nuclear matter with the two potential models result in energies that are very similar. This effect is largely due to the excellent reproduction of various partial waves for the proton-neutron channel, in particular the ${ }^{3} S_{1}$ partial wave [77. However, for pure neutron matter we see a clear deviation starting at Fermi momenta $k_{F}=1.4 \mathrm{fm}^{-1}$. To better understand this behavior, we have singled out two partial waves, namely the ${ }^{1} S_{0}$ and the ${ }^{3} P_{0}$ partial waves. The results for the potential energies per particle are shown in Figs. 12 and 13 for the ${ }^{1} S_{0}$ and the ${ }^{3} P_{0}$ partial waves, respectively. We show both the Hartree-Fock potential energy and the total potential energy by adding the results from the PPHH-LAD correlations. At the NNLO level of optimization, the $P$ waves show larger deviations from the phase shifts deduced from the experimental cross sections 77, yielding a poorer agreement compared with the $\mathrm{N}^{3} \mathrm{LO}$ interaction at lab energies beyond $100 \mathrm{MeV}$ in energy. This applies in particular to the ${ }^{3} P_{0}$ and the ${ }^{3} P_{1}$ partial waves, resulting in a ${ }^{3} P_{0}$ wave which is less attractive for the NNLO optimized interaction model. The contributions from the ${ }^{3} P_{1}$ partial wave to the equation of state plays a smaller role compared with the ${ }^{3} P_{0}$ partial wave. The differences for the ${ }^{3} P_{0}$ partial wave is seen rather clearly in

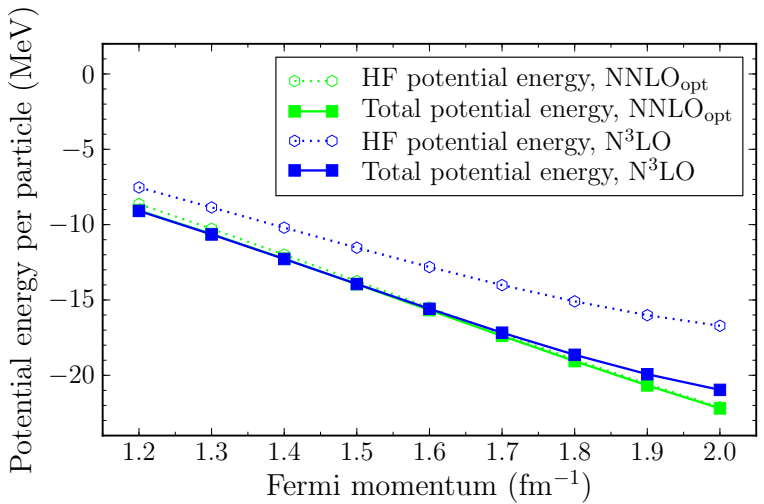

FIG. 12. (Color online) Potential energy per particle for the ${ }^{1} S_{0}$ partial wave as function of the Fermi momentum $k_{F}$ for pure neutron matter. We plot the Hartree-Fock potential energy and the total potential energy obtained by adding the correlation energies obtained from PPHH-LAD approximation with angular-averaged Pauli exclusion operators. Both potential models have been employed.

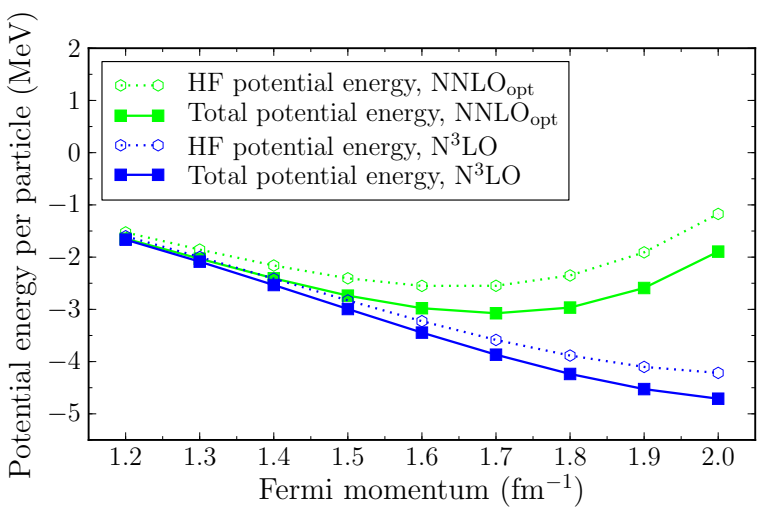

FIG. 13. (Color online) Potential energy per particle for the ${ }^{3} P_{0}$ partial wave as function of the Fermi momentum $k_{F}$ for pure neutron matter. We plot the Hartree-Fock potential energy and the total potential energy obtained by adding the correlation energies obtained from PPHH-LAD approximation with angular-averaged Pauli exclusion operators. Both potential models have been employed.

Fig. 13. The $\mathrm{N}^{3} \mathrm{LO}$ interaction results in more binding than the $\mathrm{NNLO}_{\text {opt }}$ interaction model for this particular partial wave. This applies both to the Hartree-Fock potential energy and to the final potential energy that includes correlations. The discrepancy that arises from this partial wave is the main reason behind the more repulsive equation of state obtained with the $\mathrm{NNLO}_{\mathrm{opt}}$ interaction. It will thus be interesting to see whether an optimization with respect to the experimental cross section at both the NNLO and $\mathrm{N}^{3} \mathrm{LO}$ levels will bring the results for pure neutron matter closer to the results obtained with the $\mathrm{N}^{3} \mathrm{LO}$ interaction of Ref. 64. These 
results will be presented in Ref. [77. It is interesting to note also that the Hartree-Fock potential energies for the ${ }^{1} S_{0}$ channel are rather similar to the fully correlated potential energy with the $\mathrm{NNLO}_{\text {opt }}$ interaction. This is also in line with our analysis from nuclear structure of Ref. 63, indicating that this interaction is rather soft at the two-body level. Both interaction models yield negligible differences for the full potential energy for the ${ }^{1} S_{0}$ partial wave. In summary, the poorer reproduction of the phase shifts for two selected $P$-waves, lead to a more repulsive equation of state for pure neutron matter with the newly optimized $\mathrm{NNLO}_{\text {opt }}$ interaction. Whether threebody forces or more complicated correlations beyond the CCD approximation employed here, will improve the situation, remains however to be explored.

Finally, we present our results for the symmetry energy in Fig. 14. The symmetry energy $S$ is defined as the difference between the binding energies of pure neutron matter and symmetric nuclear matter, that is

$$
S=\left(E_{\mathrm{pnm}}-E_{\mathrm{snm}}\right) / A,
$$

where $E_{\mathrm{pnm}} / A$ and $E_{\mathrm{snm}} / A$ are the binding energies per particle for pure neutron matter and symmetric nuclear matter, respectively. The behavior of the symmetry energy at high densities is important for the understanding of several physical properties and processes of neutron stars (see [78 80, and references therein). In Fig. 14 , the symmetry energy is plotted as a function of nucleon density, as obtained from a PPHH-LAD calculation with exact Pauli exclusion operators. The symmetry energies are calculated with both the $\mathrm{N}^{3} \mathrm{LO}$ and $\mathrm{NNLO}_{\text {opt }}$ twobody interactions. The symmetry energy obtained with the $\mathrm{N}^{3} \mathrm{LO}$ interaction is slightly larger than what was reported in Refs. 81, 82, where the calculations were done with BHF theory using the CD-Bonn interaction. At densities lower than $0.1 \mathrm{fm}^{-1}$, the two interaction models give almost the same symmetry energy. However, above the saturation density, the difference between the two models increases as a function of density. As seen from Fig. 14, the $\mathrm{NNLO}_{\text {opt }}$ interaction gives significantly larger symmetry energies than the $\mathrm{N}^{3} \mathrm{LO}$ interaction at high densities. Such a large deviation between the two different two-body interactions is possible since the nuclear interactions are fitted to phase shifts for laboratory energies only up to $290 \mathrm{MeV}$ [63, 64].

\section{CONCLUSIONS}

We have studied infinite nuclear and neutron matter using a coupled-cluster ladder approximation, where the equations were derived from the coupled-cluster doubles approximation. In the coupled ladder approximation, particle-hole and non-linear diagrams were neglected from the coupled-cluster doubles amplitude equations. Our approach can be seen as a first step in implementing CC theory for infinite matter. The coupled

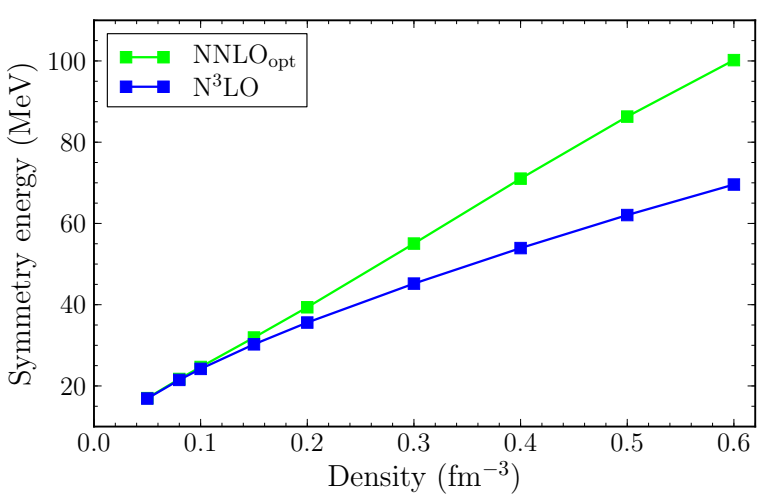

FIG. 14. (Color online) Symmetry energy as function of density, calculated in the PPHH-LAD approximation with exact Pauli exclusion operators. The empirical saturation density of symmetric nuclear matter is approximately $0.17 \mathrm{fm}^{-3}[83$.

ladder equations consist of particle-particle and hole-hole ladder diagrams which are coupled together. As we have shown, this method is closely related to the commonly used Brueckner-Hartree-Fock approximation.

We have derived coupled ladder equations both with exact and angular-averaged Pauli exclusion operators, following the approach introduced by Suzuki et al. for the BHF approximation [15. In all calculations we have used angular-averaged input momenta for the single-particle energies. Our method was applied numerically to both symmetric nuclear matter and pure neutron matter systems. The ladder approximations for symmetric nuclear matter were found to give less binding than the BHF approximation. In symmetric nuclear matter, the contribution from the hole-hole ladder diagrams was found to be smaller than the error due to angular-average approximations for the Pauli exclusion operators. Generally, symmetric nuclear matter calculations with exact Pauli exclusion operators gave more binding than calculations with angular-averaged Pauli operators. This behavior is in agreement with observations made with the BHF method [14, 15]. The binding energy per particle of pure neutron matter was found to be less sensitive to the Pauli exclusion operator approximation than what was the case for symmetric nuclear matter.

The ladder approximations were applied to infinite neutron and nuclear matter using two different chiral two-body interactions. An optimized next-to-next-toleading order (NNLO) interaction 63] was compared with the next-to-next-to-next-to-leading order $\left(\mathrm{N}^{3} \mathrm{LO}\right)$ interaction of Entem and Machleidt [64. In symmetric nuclear matter, we found that the two interaction models gave similar binding energies. As was shown in Ref. 63], the optimized NNLO interaction gives more repulsion in neutron matter compared to the $\mathrm{N}^{3} \mathrm{LO}$ two-body interaction. In the present work, we showed that the increased repulsion obtained with the NNLO interaction is due to differences in the ${ }^{3} P_{0}$ and ${ }^{3} P_{1}$ partial waves. We also cal- 
culated symmetry energies with the $\mathrm{N}^{3} \mathrm{LO}$ and optimized NNLO two-body interactions.

\section{ACKNOWLEDGMENTS}

We thank Scott Bogner, Boris Carlsson, Gustav Jansen, Øyvind Jensen, Simen Kvaal, and Thomas Pa- penbrock for several discussions. This work was supported by the Research Council of Norway under contract ISP-Fysikk/216699; by the Office of Nuclear Physics, U.S. Department of Energy (Oak Ridge National Laboratory), under DE-FG02-96ER40963 (University of Tennessee), and DE-SC0008499 (NUCLEI SciDAC collaboration). This research used computational resources of the Notur project in Norway.
[1] B. D. Day, Rev. Mod. Phys. 50, 495 (1978)

[2] B. D. Day and G. Zabolitzky, Nucl. Phys. A 366, 221 (1981)

[3] A. Akmal and V. R. Pandharipande, Phys. Rev. C 56, 2261 (1997)

[4] W. Dickhoff and C. Barbieri, Prog. Part. and Nucl. Phys. 52, 377 (2004)

[5] S. Gandolfi, F. Pederiva, S. Fantoni, and K. E. Schmidt, Phys. Rev. Lett. 98, 102503 (2007)

[6] Z. H. Li, U. Lombardo, H.-J. Schulze, W. Zuo, L. W. Chen, and H. R. Ma, Phys. Rev. C 74, 047304 (2006)

[7] A. Lovato, O. Benhar, S. Fantoni, A. Y. Illarionov, and K. E. Schmidt, Phys. Rev. C 83, 054003 (2011)

[8] A. Carbone, A. Polls, and A. Rios, Phys. Rev. C 88, $044302(2013)$

[9] K. A. Brueckner, Phys. Rev. 100, 36 (1955)

[10] B. D. Day, Rev. Mod. Phys. 39, 719 (1967)

[11] K. A. Brueckner and C. A. Levinson, Phys. Rev. 97, 1344 (1955)

[12] M. I. Haftel and F. Tabakin, Nucl. Phys. A 158, 1 (1970)

[13] H. Q. Song, M. Baldo, G. Giansiracusa, and U. Lombardo, Phys. Rev. Lett. 81, 1584 (1998)

[14] E. Schiller, H. Müther, and P. Czerski, Phys. Rev. C 60, 059901(E) (1999)

[15] K. Suzuki, R. Okamoto, M. Kohno, and S. Nagata, Nucl. Phys. A 665, 92 (2000)

[16] M. Baldo, A. Polls, A. Rios, H.-J. Schulze, and I. Vidaña, Phys. Rev. C 86, 064001 (2012)

[17] R. Rajaraman and H. A. Bethe, Rev. Mod. Phys. 39, 745 (1967)

[18] B. D. Day, Phys. Rev. C 24, 1203 (1981)

[19] V. Somà and P. Bozek, Phys. Rev. C 78, 054003 (2008)

[20] I. Vidaña, C. Providência, A. Polls, and A. Rios, Phys. Rev. C 80, 045806 (2009)

[21] K. Hebeler, S. K. Bogner, R. J. Furnstahl, A. Nogga, and A. Schwenk, Phys. Rev. C 83, 031301 (2011)

[22] A. W. Steiner and S. Gandolfi, Phys. Rev. Lett. 108, $081102(2012)$

[23] K. Hebeler and R. J. Furnstahl, Phys. Rev. C 87, 031302 (2013)

[24] I. Tews, T. Krüger, K. Hebeler, and A. Schwenk, Phys. Rev. Lett. 110, 032504 (2013)

[25] T. Krüger, I. Tews, K. Hebeler, and A. Schwenk, Phys. Rev. C 88, 025802 (2013)

[26] F. Coester, Nucl. Phys. 7, 421 (1958)

[27] F. Coester and H. Kümmel, Nucl. Phys. 17, 477 (1960)

[28] J. Čížek, J. Chem. Phys. 45, 4256 (1966)

[29] J. Č́žek, "On the Use of the Cluster Expansion and the Technique of Diagrams in Calculations of Correlation Effects in Atoms and Molecules," in Advances in Chemical Physics (John Wiley \& Sons, Inc., 2007) p. 35
[30] J. Č́ižek and J. Paldus, Int. J. Quant. Chem. 5, 359 (1971)

[31] R. Bartlett and M. Musiał, Rev. Mod. Phys. 79, 291 (2007)

[32] I. Shavitt and R. J. Bartlett, Many-Body Methods in Chemistry and Physics (Cambridge University Press, 2009)

[33] T. D. Crawford and H. F. Schaefer, Rev. Comp. Chem. 14, 33 (2000)

[34] F. E. Harris, H. J. Monkhorst, and D. L. Freeman, Algebraic and diagrammatic methods in many-fermion theory (Oxford University Press, 1992)

[35] J. H. Heisenberg and B. Mihaila, Phys. Rev. C 59, 1440 (1999)

[36] B. Mihaila and J. H. Heisenberg, Phys. Rev. C 61, 054309 (2000)

[37] D. J. Dean and M. Hjorth-Jensen, Phys. Rev. C 69, $054320(2004)$

[38] J. R. Gour, P. Piecuch, M. Hjorth-Jensen, M. Włoch, and D. J. Dean, Phys. Rev. C 74, 024310 (2006)

[39] J. R. Gour, M. Horoi, P. Piecuch, and B. A. Brown, Phys. Rev. Lett. 101, 052501 (2008)

[40] G. Hagen, T. Papenbrock, D. J. Dean, and M. HjorthJensen, Phys. Rev. C 82, 034330 (2010)

[41] G. R. Jansen, M. Hjorth-Jensen, G. Hagen, and T. Papenbrock, Phys. Rev. C 83, 054306 (2011)

[42] O. Jensen, G. Hagen, T. Papenbrock, D. J. Dean, and J. S. Vaagen, Phys. Rev. C 82, 014310 (2010)

[43] R. Roth, S. Binder, K. Vobig, A. Calci, J. Langhammer, and P. Navrátil, Phys. Rev. Lett. 109, 052501 (2012)

[44] H. Kümmel, K. H. Lührmann, and J. G. Zabolitzky, Phys. Rep. 36, 1 (1978)

[45] R. F. Bishop, Theor Chim Acta 80, 95 (1991)

[46] R. Machleidt and I. Slaus, J. Phys. G: Nucl. Part. Phys. 27, R69 (2001)

[47] R. Machleidt and D. R. Entem, Phys. Rep. 503, 1 (2011)

[48] S. K. Bogner, A. Schwenk, R. J. Furnstahl, and A. Nogga, Nucl. Phys. A 763, 59 (2005)

[49] W. Manzke, Diplomarbeit, Bochum University (1974)

[50] R. F. Bishop and K. H. Lührmann, Phys. Rev. 17, 3757 (1978)

[51] D. L. Freeman, Phys. Rev. B 15, 5512 (1977)

[52] D. L. Freeman, J. Phys. C: Solid State Phys. 16, 711 (1983)

[53] W. Dickhoff, A. Faessler, and H. Müther, Nucl. Phys. A 389, 492 (1982)

[54] A. Ramos, A. Polls, and W. H. Dickhoff, Nucl. Phys. A 503, 1 (1989)

[55] P. Bozek, Eur. Phys. J. A 15, 325 (2002), ISSN 1434-6001

[56] Y. Dewulf, W. H. Dickhoff, D. Van Neck, E. R. Stoddard, and M. Waroquier, Phys. Rev. Lett. 90, 152501 (2003) 
[57] T. Frick and H. Müther, Phys. Rev. C 68, 034310 (2003)

[58] A. Rios, A. Polls, and I. Vidaña, Phys. Rev. C 79, 025802 (2009)

[59] H. Song, S. Yang, and T. Kuo, Nucl. Phys. A 462, 491 (1987)

[60] M. F. Jiang, T. T. S. Kuo, and H. Müther, Phys. Rev. C 38, 2408 (1988)

[61] L. Engvik, E. Osnes, M. Hjorth-Jensen, and T. Kuo, Nucl. Phys. A 622, 553 (1997)

[62] L.-W. Siu, J. W. Holt, T. T. S. Kuo, and G. E. Brown, Phys. Rev. C 79, 054004 (2009)

[63] A. Ekström, G. Baardsen, C. Forssén, G. Hagen, M. Hjorth-Jensen, G. R. Jansen, R. Machleidt, W. Nazarewicz, T. Papenbrock, J. Sarich, and S. M. Wild, Phys. Rev. Lett. 110, 192502 (2013)

[64] D. R. Entem and R. Machleidt, Phys. Rev. C 68, 041001(R) (2003)

[65] T. Munson, J. Sarich, S. M. Wild, S. Benson, and L. Curfman McInnes, TAO 2.0 Users Manual, Technical Memorandum ANL/MCS-TM-322 (Argonne National Laboratory, Argonne, Illinois, 2012) http://www.mcs.anl.gov/ uploads/cels/papers/TM-322.pdf

[66] J. J. MacKenzie, Phys. Rev. 179, 1002 (1969)

[67] K. A. Brueckner and J. L. Gammel, Phys. Rev. 109, 1023 (1958)

[68] C. Mahaux, P. F. Bortignon, and R. A. Broglia, Phys. Rep. 120, 1 (1985)

[69] C. Mahaux and R. Sartor, Phys. Rev. C 40, 1833 (1989)

[70] À. G. Ramos, Ph.D. thesis, University of Barcelona (1988)

[71] D. A. Varshalovich, A. N. Moskalev, and V. K. Khersonskii, Quantum Theory of Angular Momentum (World Scientific, 1988)

[72] M. Baldo, I. Bombaci, L. S. Ferreira, G. Giansiracusa, and U. Lombardo, Phys. Rev. C 43, 2605 (1991)

[73] A. Polls et al., in preparation(2013)

[74] R. B. Wiringa, V. G. J. Stoks, and R. Schiavilla, Phys. Rev. C 51, 38 (1995)

[75] B. Vonderfecht, W. Dickhoff, A. Polls, and A. Ramos, Nuclear Physics A 555, 1 (1993)

[76] D. J. Dean and M. Hjorth-Jensen, Rev. Mod. Phys. 75, $607(2003)$

[77] A. Ekström, in preparation(2013)

[78] J. M. Lattimer, Annu. Rev. Nucl. Part. Science 62, 485 (2012)

[79] B.-A. Li, Phys. Rev. Lett. 88, 192701 (2002)

[80] J. Erler, C. J. Horowitz, W. Nazarewicz, M. Rafalski, and P.-G. Reinhard, Phys. Rev. C 87, 044320 (2013)

[81] L. Engvik, M. Hjorth-Jensen, R. Machleidt, H. Müther, and A. Polls, Nucl. Phys. A 627, 85 (1997)

[82] H. Heiselberg and M. Hjorth-Jensen, Phys. Rep. 328, 237 (2000)

[83] W. D. Myers and W. J. Swiatecki, Ann. of Phys. 204, 401 (1990)

\section{Appendix A: Technical details}

\section{Relative momentum basis}

Infinite nuclear matter is defined in the thermodynamic limit, that is, the limit where the volume $\Omega$ and the number of particles $A$ approach infinity, while the density of particles $\rho \equiv A / \Omega$ is kept constant. At the limit when $\Omega$ approaches infinity, the sums over momenta can be replaced by integrals:

$$
\sum_{\mathbf{k}} \rightarrow \frac{\Omega}{(2 \pi)^{3}} \int d \mathbf{k} .
$$

We will in the following replace all sums over momenta by integrals according to Eq. A1.

Taking the limit $\Omega \rightarrow \infty$, the ladder amplitude equations (8) may be written in laboratory momentum coordinates as

$$
\begin{aligned}
0 & =\left\langle\mathbf{k}_{a} \mathbf{k}_{b}|v| \mathbf{k}_{i} \mathbf{k}_{j}\right\rangle \\
& +\left(\varepsilon\left(\mathbf{k}_{a}\right)+\varepsilon\left(\mathbf{k}_{b}\right)-\varepsilon\left(\mathbf{k}_{i}\right)-\varepsilon\left(\mathbf{k}_{j}\right)\right)\left\langle\mathbf{k}_{a} \mathbf{k}_{b}|t| \mathbf{k}_{i} \mathbf{k}_{j}\right\rangle \\
& +\frac{1}{2}\left(\frac{\Omega}{(2 \pi)^{3}}\right)^{2} \int d \mathbf{k}_{k} \int d \mathbf{k}_{l}\left\langle\mathbf{k}_{a} \mathbf{k}_{b}|t| \mathbf{k}_{k} \mathbf{k}_{l}\right\rangle\left\langle\mathbf{k}_{k} \mathbf{k}_{l}|v| \mathbf{k}_{i} \mathbf{k}_{j}\right\rangle \\
& \times \theta\left(k_{F}-\left|\mathbf{k}_{k}\right|\right) \theta\left(k_{F}-\left|\mathbf{k}_{l}\right|\right) \\
& +\frac{1}{2}\left(\frac{\Omega}{(2 \pi)^{3}}\right)^{2} \int d \mathbf{k}_{c} \int \mathbf{k}_{d}\left\langle\mathbf{k}_{a} \mathbf{k}_{b}|v| \mathbf{k}_{c} \mathbf{k}_{d}\right\rangle\left\langle\mathbf{k}_{c} \mathbf{k}_{d}|t| \mathbf{k}_{i} \mathbf{k}_{j}\right\rangle \\
& \times \theta\left(\left|\mathbf{k}_{c}\right|-k_{F}\right) \theta\left(\left|\mathbf{k}_{d}\right|-k_{F}\right),
\end{aligned}
$$

where $\theta(x)$ is the Heaviside step function and we have used the definition $\varepsilon(\mathbf{k}) \equiv\langle\mathbf{k}|f| \mathbf{k}\rangle$. Later, we will refer to $\varepsilon(\mathbf{k})$ as the single-particle energy.

We define the relative and center-of-mass (RCM) momentum coordinates as

$$
\begin{aligned}
\mathbf{k} & =\left(\mathbf{k}_{i}-\mathbf{k}_{j}\right) / 2, & \mathbf{K} & =\mathbf{k}_{i}+\mathbf{k}_{j} \\
\mathbf{k}^{\prime} & =\left(\mathbf{k}_{a}-\mathbf{k}_{b}\right) / 2, & \mathbf{K}^{\prime} & =\mathbf{k}_{a}+\mathbf{k}_{b} \\
\mathbf{h} & =\left(\mathbf{k}_{k}-\mathbf{k}_{l}\right) / 2, & \mathbf{H} & =\mathbf{k}_{k}+\mathbf{k}_{l} \\
\mathbf{p} & =\left(\mathbf{k}_{c}-\mathbf{k}_{d}\right) / 2, & \mathbf{P} & =\mathbf{k}_{c}+\mathbf{k}_{d},
\end{aligned}
$$

where $i, j, k, l$ denote single-particle states occupied and $a, b, c, d$ states unoccupied in the uncorrelated Fermi vacuum state. Transforming to RCM coordinates, the PPHH-LAD equations become

$$
\begin{aligned}
0 & =\left\langle\mathbf{k}^{\prime}|v| \mathbf{k}\right\rangle \\
& +\left(\varepsilon\left(\left|\mathbf{k}^{\prime}+\mathbf{K} / 2\right|\right)+\varepsilon\left(\left|-\mathbf{k}^{\prime}+\mathbf{K} / 2\right|\right)\right. \\
& -\varepsilon(|\mathbf{k}-\mathbf{K} / 2|)-\varepsilon(|-\mathbf{k}+\mathbf{K} / 2|))\left\langle\mathbf{k}^{\prime}|t| \mathbf{k}\right\rangle \\
& +\frac{1}{2} \int d \mathbf{h}\left\langle\mathbf{k}^{\prime}|t(\mathbf{K})| \mathbf{h}\right\rangle\langle\mathbf{h}|v| \mathbf{k}\rangle \\
& \times \theta\left(k_{F}-|\mathbf{h}+\mathbf{K} / 2|\right) \theta\left(k_{F}-|-\mathbf{h}+\mathbf{K} / 2|\right) \\
& +\frac{1}{2} \int d \mathbf{p}\left\langle\mathbf{k}^{\prime}|v| \mathbf{p}\right\rangle\langle\mathbf{p}|t(\mathbf{K})| \mathbf{k}\rangle \\
& \times \theta\left(|\mathbf{p}+\mathbf{K} / 2|-k_{F}\right) \theta\left(|-\mathbf{p}+\mathbf{K} / 2|-k_{F}\right),
\end{aligned}
$$

where the relation

$$
\left\langle\mathbf{k}_{p} \mathbf{k}_{q}|v| \mathbf{k}_{r} \mathbf{k}_{s}\right\rangle=\frac{(2 \pi)^{3}}{\Omega}\left\langle\mathbf{k}|v| \mathbf{k}^{\prime}\right\rangle \delta_{\mathbf{K K}^{\prime}}
$$

has been used. The expressions $(9)$ and $(10)$ for the reference and correlation energies, respectively, can be transformed to RCM coordinates in a similar way as is shown here for the ladder amplitude equations. 
Due to the isotropy of nuclear matter, we assume that the single-particle energy $\varepsilon\left(\mathbf{k}_{p}\right)$ depends only on the absolute value of the argument $\mathbf{k}_{p}$ [54, 70]. In laboratory frame momentum coordinates, the single-particle energy is then

$$
\varepsilon\left(\left|\mathbf{k}_{p}\right|\right)=\frac{\hbar^{2} k_{p}^{2}}{2 m}+U\left(\left|\mathbf{k}_{p}\right|\right)
$$

where

$$
U\left(\left|\mathbf{k}_{p}\right|\right)=\frac{\Omega}{(2 \pi)^{3}} \sum_{m_{s} m_{t}} \int d \mathbf{k}_{q}\left\langle\mathbf{k}_{p} \mathbf{k}_{q}|v| \mathbf{k}_{p} \mathbf{k}_{q}\right\rangle \theta\left(k_{F}-\left|\mathbf{k}_{q}\right|\right) .
$$

Because of the isotropy, we choose the direction such that $\mathbf{k}_{p}=\left(0,0, k_{p}\right)$. The single-particle potential energy can also be written as

$$
\begin{aligned}
U\left(\left|\mathbf{k}_{p}\right|\right)= & \sum_{m_{s} m_{t}} \int d \mathbf{k}_{q}[\langle\mathbf{p}|v| \mathbf{p}\rangle-\langle\mathbf{p}|v|-\mathbf{p}\rangle] \\
& \times \theta\left(k_{F}-|-\mathbf{p}+\mathbf{P} / 2|\right),
\end{aligned}
$$

where $\mathbf{p}$ and $\mathbf{P}$ are relative and center-of-mass momentum coordinates defined by $\mathbf{k}_{p}$ and $\mathbf{k}_{q}$, as in Eq. A3.

\section{Momentum and angular momentum basis}

In this work, we assume that every interaction matrix element

$$
\left\langle k^{\prime}\left(l^{\prime} S\right) \mathcal{J} m_{\mathcal{J}} M_{T}|v| k(l S) \mathcal{J} m_{\mathcal{J}} M_{T}\right\rangle
$$

and every $t$-amplitude matrix element is multiplied by a factor

$$
\mathcal{A}^{l^{\prime} l S M_{T}}= \begin{cases}1+(-1)^{l+l^{\prime}}, & \text { if } M_{T}=0, \\ \frac{1}{2}\left(1-(-1)^{l+S+1}\right) & \\ \times\left(1-(-1)^{l^{\prime}+S+1}\right), & \text { if }\left|M_{T}\right|=1,\end{cases}
$$

which ensures antisymmetry and conservation of parity.

Using exact Pauli exclusion operators, the ladder $t$ amplitude equations (A4) can be rewritten in the coupled angular momentum basis as

$$
\begin{aligned}
& \Delta \varepsilon\left(\mathbf{k}, \mathbf{k}^{\prime}, \mathbf{K}\right)\left\langle k^{\prime}\left(l^{\prime} S\right) \mathcal{J}^{\prime} m_{\mathcal{J}^{\prime}} M_{T}\left|t\left(\mathbf{K}, \hat{\mathbf{k}}, \hat{\mathbf{k}}^{\prime}\right)\right| k(l S) \mathcal{J} m_{\mathcal{J}} M_{T}\right\rangle \\
& =\left\langle k^{\prime}\left(l^{\prime} S\right) \mathcal{J}^{\prime} m_{\mathcal{J}^{\prime}} M_{T}|v| k(l S) \mathcal{J} m_{\mathcal{J}} M_{T}\right\rangle \delta_{\mathcal{J}} \mathcal{J}^{\prime} \delta_{m_{\mathcal{J}} m_{\mathcal{J}^{\prime}}} \\
& +\frac{1}{2} \sum_{\mathcal{J}^{\prime \prime} m_{\mathcal{J}^{\prime \prime}}} \sum_{l^{\prime \prime} l^{\prime \prime \prime}} \int_{0}^{k_{F}} h^{2} d h \\
& \times\left\langle k^{\prime}\left(l^{\prime} S\right) \mathcal{J}^{\prime} m_{\mathcal{J}^{\prime}} M_{T}\left|t\left(\mathbf{K}, \hat{\mathbf{k}}, \hat{\mathbf{k}}^{\prime}\right)\right| h\left(l^{\prime \prime} S\right) \mathcal{J}^{\prime \prime} m_{\mathcal{J}^{\prime \prime}} M_{T}\right\rangle \\
& \times\left\langle h\left(l^{\prime \prime \prime} S\right) \mathcal{J}_{\mathcal{J}^{\prime}} M_{T}|v| k(l S) \mathcal{J} m_{\mathcal{J}} M_{T}\right\rangle \\
& \times Q_{h h}\left(l^{\prime \prime} \mathcal{J}^{\prime \prime} m_{\mathcal{J}^{\prime \prime}}, l^{\prime \prime \prime} \mathcal{J} m_{\mathcal{J}} ; S M_{T} h K \theta_{K} \phi_{K}\right) \\
& +\frac{1}{2} \sum_{\mathcal{J}^{\prime \prime} m_{\mathcal{J}^{\prime \prime}} l_{l^{\prime \prime} l^{\prime \prime \prime}} \int_{0}^{\infty} p^{2} d p} \times\left\langle k^{\prime}\left(l^{\prime} S\right) \mathcal{J}^{\prime} m_{\mathcal{J}^{\prime}} M_{T}|v| p\left(l^{\prime \prime} S\right) \mathcal{J}^{\prime} m_{\mathcal{J}^{\prime}} M_{T}\right\rangle \\
& \times\left\langle p\left(l^{\prime \prime \prime} S\right) \mathcal{J}^{\prime \prime} m_{\mathcal{J}^{\prime \prime}} M_{T}\left|t\left(\mathbf{K}, \hat{\mathbf{k}}, \hat{\mathbf{k}}^{\prime}\right)\right| k(l S) \mathcal{J} m_{\mathcal{J}} M_{T}\right\rangle \\
& \times Q_{p p}\left(l^{\prime \prime} \mathcal{J}^{\prime} m_{\mathcal{J}^{\prime}}, l^{\prime \prime \prime} \mathcal{J}^{\prime \prime} m_{\mathcal{J}^{\prime \prime}} ; S M_{T} K \theta_{K} \phi_{K}\right), \quad(\mathrm{A} 10)
\end{aligned}
$$

where $\left|(l S) \mathcal{J} m_{\mathcal{J}}\right\rangle$ denotes a vector where $l$ and $S$ are coupled to $\mathcal{J}$. In Eq. A10 we have introduced the shorthand notation

$$
\begin{aligned}
\Delta \varepsilon\left(\mathbf{k}, \mathbf{k}^{\prime}, \mathbf{K}\right) & \equiv \varepsilon(|\mathbf{k}+\mathbf{K} / 2|)+\varepsilon(|-\mathbf{k}+\mathbf{K} / 2|) \\
& -\varepsilon\left(\left|\mathbf{k}^{\prime}+\mathbf{K} / 2\right|\right)-\varepsilon\left(\left|-\mathbf{k}^{\prime}+\mathbf{K} / 2\right|\right)
\end{aligned}
$$

for the energy denominator.

The sum of the single-particle energies corresponding to two hole states can be expressed in terms of RCM coordinates as

$$
\begin{aligned}
\varepsilon\left(\left|\mathbf{k}_{i}\right|\right)+\varepsilon\left(\left|\mathbf{k}_{j}\right|\right)= & \varepsilon(|\mathbf{k}+\mathbf{K} / 2|)+\varepsilon(|-\mathbf{k}+\mathbf{K} / 2|) \\
= & \frac{\hbar^{2} k^{2}}{m}+\frac{\hbar^{2} K^{2}}{4 m} \\
& +U^{M_{T},+}(|\mathbf{k}+\mathbf{K} / 2|) \\
& +U^{M_{T},-}(|-\mathbf{k}+\mathbf{K} / 2|),
\end{aligned}
$$

where

$$
\begin{aligned}
U^{M_{T}, \pm}\left(\left|\mathbf{k}_{p}\right|\right)= & \frac{1}{4} \sum_{\mathcal{J} S l}(2 \mathcal{J}+1) \int_{0}^{k_{F}} d k_{q} k_{q}^{2} \int_{-1}^{1} d \cos \theta_{\mathbf{k}_{q}} \\
& \times \mathcal{B}^{M_{T}, \pm}\langle p \mathcal{J} l S|v| p \mathcal{J} l S\rangle,
\end{aligned}
$$

and the variable $p=\left|\mathbf{k}_{p}-\mathbf{k}_{q}\right| / 2$. The antisymmetrization operator $\mathcal{B}^{M_{T}, \pm}$ is defined through the relation

$$
\begin{aligned}
& \mathcal{B}^{M_{T}, \pm}\left\langle p \mathcal{J} l S|v| p \mathcal{J} l^{\prime} S\right\rangle=\left\langle p \mathcal{J} l S\left|v\left(M_{T}^{\prime}=0\right)\right| p \mathcal{J} l^{\prime} S\right\rangle \\
& +\left(1-(-1)^{l^{\prime}+S^{\prime}+1}\right) \\
& \times\left\langle p \mathcal{J} l S\left|v\left(M_{T}^{\prime}=M_{T} \pm \delta_{M_{T}, 0}\right)\right| p \mathcal{J} l^{\prime} S\right\rangle
\end{aligned}
$$

for symmetric nuclear matter and the relation

$$
\begin{aligned}
& \mathcal{B}^{M_{T}, \pm}\left\langle p \mathcal{J} l S|v| p \mathcal{J} l^{\prime} S\right\rangle=\left(1-(-1)^{l^{\prime}+S^{\prime}+1}\right) \\
& \times\left\langle p \mathcal{J} l S\left|v\left(M_{T}^{\prime}=1\right)\right| p \mathcal{J} l^{\prime} S\right\rangle
\end{aligned}
$$

for pure neutron matter. The expressions of $\mathbf{k}$ and $\mathbf{K}$ are given in Eq. A3). The sum of single-particle energies corresponding to two particle states, $\varepsilon\left(\mathbf{k}_{a}\right)+\varepsilon\left(\mathbf{k}_{b}\right)$, is calculated in the same way. We define the angularaveraged energy denominator as

$$
\Delta \tilde{\varepsilon}\left(k, k^{\prime}, K\right) \equiv \varepsilon\left(\overline{k_{i}}\right)+\varepsilon\left(\overline{k_{j}}\right)-\varepsilon\left(\overline{k_{a}}\right)-\varepsilon\left(\overline{k_{b}}\right),
$$

where $\overline{k_{p}}$ for $p=i, j, a, b$ are angular-averaged input momenta defined in Eq. (17). Observe that the energy denominator is assumed to be a function of the two-particle isospin projection $M_{T}$.

The correlation energy can be written in the partial 
wave expansion as

$$
\begin{aligned}
& \Delta E_{C C D} / A=\frac{3 C}{64 \pi k_{F}^{3}} \sum_{\mathcal{J} m_{\mathcal{J}}} \sum_{\mathcal{J}^{\prime \prime} m_{\mathcal{J}^{\prime \prime}}} \sum_{\mathcal{J}^{\prime \prime \prime} m_{\mathcal{J}^{\prime \prime \prime}}} \sum_{S M_{T}} \sum_{l l^{\prime} l^{\prime \prime} l^{\prime \prime \prime}} \\
& \times \int_{0}^{\sqrt{k_{F}^{2}-K^{2} / 4}} k^{2} d k \int_{\sqrt{k_{F}^{2}-K^{2} / 4}}^{\infty} d k^{\prime} \int_{0}^{2 k_{F}} K^{2} d K \\
& \times \int_{-1}^{1} d \cos \theta_{K} \int_{0}^{2 \pi} d \phi_{K}\left\langle k(l S) \mathcal{J} M_{T}|v| k^{\prime}\left(l^{\prime} S\right) \mathcal{J} M_{T}\right\rangle \\
& \times \hat{Q}_{h h}\left(l^{\prime \prime \prime} \mathcal{J}^{\prime \prime \prime} m_{\mathcal{J}^{\prime \prime \prime}}, l \mathcal{J}_{m_{\mathcal{J}}} ; S M_{T} k K \theta_{K} \phi_{K}\right) \\
& \times \hat{Q}_{p p}\left(l^{\prime} \mathcal{J}_{m_{\mathcal{J}}, l^{\prime \prime} \mathcal{J}^{\prime \prime} m_{\mathcal{J}} ; S} ; M_{T} k^{\prime} K \theta_{K} \phi_{K}\right) \\
& \times\left\langle k^{\prime}\left(l^{\prime \prime} S\right) \mathcal{J}^{\prime \prime} m_{\mathcal{J}^{\prime \prime}} M_{T}\left|t\left(\mathbf{K}, \hat{\mathbf{k}}, \hat{\mathbf{k}}^{\prime}\right)\right| k\left(l^{\prime \prime \prime} S\right) \mathcal{J}^{\prime \prime \prime} m_{\mathcal{J}^{\prime \prime \prime}} M_{T}\right\rangle
\end{aligned}
$$

where the Pauli operators $\hat{Q}_{h h}$ and $\hat{Q}_{p p}$ are defined in Eqs. 20 and 21. Here we use the notations $\hat{Q}_{h h}$ and $\hat{Q}_{p p}$ instead of $Q_{h h}$ and $Q_{p p}$ to emphasize that these are integral operators that operate on the $t$-amplitude matrix. The $t$-amplitude depends on $\hat{\mathbf{k}} \equiv\left(\theta_{\mathbf{k}}, \phi_{\mathbf{k}}\right)$ and $\hat{\mathbf{k}}^{\prime} \equiv$ $\left(\theta_{\mathbf{k}^{\prime}}, \phi_{\mathbf{k}^{\prime}}\right)$ through the energy denominator $\Delta \varepsilon\left(\mathbf{k}, \mathbf{k}^{\prime}, \mathbf{K}\right)$, and the closed-form expression 22 can therefore generally not be used in the energy equation A17.

When the ladder equations are written in the coupled partial wave basis, it is possible to calculate the $t$-amplitude matrix for only one angular direction of the $\mathrm{CM}$ momentum $\mathbf{K}$, and then obtain the other matrix elements by performing a rotation [15]. Using the same technique as Suzuki et al., an amplitude matrix with a general CM momentum vector can be written

$$
\begin{aligned}
& \left\langle k^{\prime}\left(l^{\prime \prime} S\right) \mathcal{J}^{\prime \prime} m_{\mathcal{J}^{\prime \prime}}|t(\mathbf{K})| k\left(l^{\prime \prime \prime} S\right) \mathcal{J}^{\prime \prime \prime} m_{\mathcal{J}^{\prime \prime \prime}}\right\rangle \\
& \left.=\sum_{m_{\mathcal{J}} m_{\mathcal{J}^{\prime}}} D_{m_{\mathcal{J}^{\prime \prime}} m_{\mathcal{J}}}^{\mathcal{J}^{\prime \prime}}\left(\phi_{K}, \theta_{K}, 0\right) D_{m_{\mathcal{J}^{\prime \prime \prime}} m_{\mathcal{J}^{\prime}}}^{\mathcal{J}^{\prime \prime \prime}}{ } \phi_{K}, \theta_{K}, 0\right) \\
& \times\left\langle k^{\prime}\left(l^{\prime \prime} S\right) \mathcal{J}^{\prime \prime} m_{\mathcal{J}}|t(K)| k\left(l^{\prime \prime \prime} S\right) \mathcal{J}^{\prime \prime \prime} m_{\mathcal{J}^{\prime}}\right\rangle
\end{aligned}
$$

where $D_{m_{\mathcal{J}} m_{\mathcal{J}^{\prime}}}^{\mathcal{J}}(\alpha, \beta, \gamma)$ is the Wigner $D$-function and $\alpha$, $\beta$, and $\gamma$ are Euler angles, defined in for example Ref. [71. Eq. A18 can be used to obtain the correlation energy expression 18 from Eq. A17. 